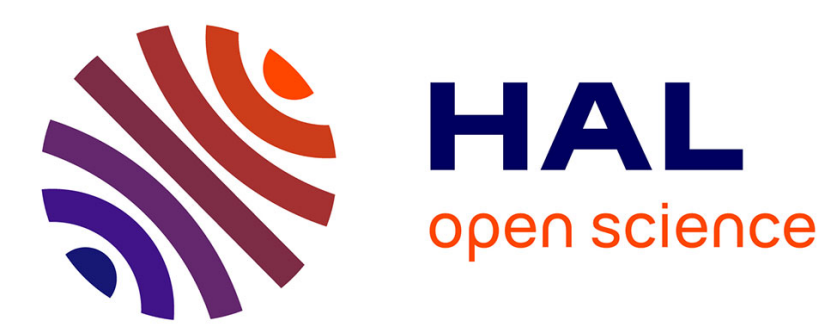

\title{
A Locking-Free Approximation of Curved Rods by Straight Beam Elements
}

Dominique Chapelle

\section{To cite this version:}

Dominique Chapelle. A Locking-Free Approximation of Curved Rods by Straight Beam Elements. [Research Report] RR-2733, INRIA. 1995. inria-00073961

\section{HAL Id: inria-00073961 https://hal.inria.fr/inria-00073961}

Submitted on 24 May 2006

HAL is a multi-disciplinary open access archive for the deposit and dissemination of scientific research documents, whether they are published or not. The documents may come from teaching and research institutions in France or abroad, or from public or private research centers.
L'archive ouverte pluridisciplinaire HAL, est destinée au dépôt et à la diffusion de documents scientifiques de niveau recherche, publiés ou non, émanant des établissements d'enseignement et de recherche français ou étrangers, des laboratoires publics ou privés. 


\section{A locking-free approximation of curved rods by straight beam elements}

Dominique Chapelle

\section{$\mathbf{N}^{\circ} \mathbf{2 7 3 3}$}

Novembre 1995

PROGRAMME 6 



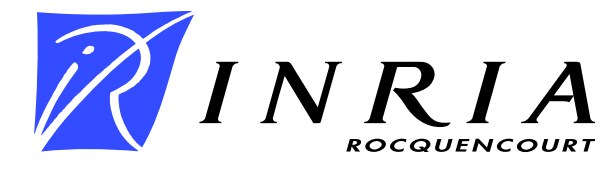

\title{
A locking-free approximation of curved rods by straight beam elements
}

\author{
Dominique Chapelle \\ Programme 6 - Calcul scientifique, modélisation et logiciel numérique \\ Projet Modulef and L.C.P.C., UMR 113 LCPC/CNRS - 2, allée Kepler, \\ 77420 Champs-sur-Marne, e.mail: chapelle@inrets.fr
}

Rapport de recherche $\mathrm{n}^{\circ} 2733$ - Novembre 1995 - 34 pages

\begin{abstract}
We consider an elastic model for a curved rod with arbitrary threedimensional geometry, incorporating shear and membrane as well as bending and torsion effects. We define an approximation procedure based on a discretization by linear Timoshenko beam elements. Introducing an equivalent mixed problem, we establish optimal error estimates independent of the thickness, thereby proving that shear and membrane locking is avoided. The approximation scheme is tested on specific examples and the numerical results confirm the estimates obtained by theory.
\end{abstract}

(Résumé : tsvp)

This work is part of the Human Capital and Mobility Program "Shells: Mathematical Modeling and Analysis, Scientific Computing" of the Commission of the European Communities (Contract \# ERBCHRXCT940536) 


\section{Approximation sans verrouillage d'un modèle de poutre courbe par des éléments de poutre droite}

Résumé : On s'intéresse à un modèle de poutre courbe non-nécessairement plane qui prend en compte des déformations de flexion, torsion, membrane et cisaillement. On en définit un schéma d'approximation en substituant à cette poutre un assemblage d'éléments finis linéaires de poutre droite de type Timoshenko. En utilisant un problème mixte équivalent, on démontre une estimation d'erreur optimale et indépendante de l'épaisseur, ce qui prouve que la méthode est exempte de verrouillage de cisaillement ou de membrane. Le schéma est ensuite testé sur des exemples et les résultats numériques obtenus sont en accord avec la théorie. 


\section{Introduction}

Models of curved rods, like other thin structures such as beams, plates and shells, are known to give rise to numerical locking when the thickness is very small compared to other dimensions. In practice, this phenomenon often reveals itself through unacceptably small numerical results (see e.g. [4]). From a numerical analysis perspective, a characteristic symptom is that basic a priori error estimates for these formulations incorporate a dependency on the thickness of the structure by which they degenerate when this parameter approaches zero. The mathematical litterature on the locking of models of thin structures is far too developped for us to review here, and we instead refer to the following texts and the references therein: $[1,7]$ regarding beams and arches, [6] for plates and [14, 2] for shells.

Locking for the Timoshenko beam model was extensively studied in [1], where the shear term was identified as the source of the difficulty and was treated by mixed methods or equivalent reduced-integration methods in order to obtain error estimates independent of the thickness. Similar methods were used in the case of a circular arch formulation without shear in [10], for locking originating from the membrane term. Significantly different was the approach in [12] where a model of circular arch containing both membrane and shear effects was analysed using a HellingerReissner formulation and a numerical scheme based on a mixed Petrov-Galerkin method. Circular arches with shear and membrane strains were also considered in [15], where a mixed formulation closer to that of [1] was employed. These results were later extended by [3] to rods with arbitrary three-dimensional geometry and response.

The perspective of this paper is different. We start from a three-dimensional rod formulation similar to that presented in [3], but we use a discretization by straight beam finite elements as an approximation procedure. This idea, which seems to have been exploited very early in engineering practice [13], was substantiated by several theoretical studies for planar arches where convergence results were established $[9,8,5]$. However, as they considered the thickness as a fixed datum, these works did not investigate the question of the sensitivity of convergence with respect to the thickness parameter.

The only existing theoretical analysis pertaining to the locking-free property of

such an approximation seems to be that in [11]. It considers a standard planar arch formulation without shear discretized by Bernoulli beam elements, and establishes 
optimal rates of convergence independent of the thickness in the case where the structure is clamped at one end and free at the other. To prove this, a mixed method equivalent to the displacement-based scheme is introduced and analysed. Yet, in the course of the argument, the assumption on the boundary conditions appears as an important restriction that crucially enters in the proof which does not hold in different configurations (such as a structure clamped at both ends).

Our analysis involves no such restriction. It also differs from [11] in that we consider general three-dimensional geometries and a formulation including shear effects so that we naturally employ Timoshenko instead of Bernoulli beam elements, with simple linear shape functions. In Section 2, we introduce the notation, give definitions concerning the geometry as well as the variational model, and establish preliminary results pertaining to the exact solution. Then in Section 3 we describe the approximation procedure, define an equivalent mixed method and prove that optimal error estimates hold independently of the thickness parameter. In addition we obtain improved error estimates in $L^{2}$-norm. Finally, in Section 4 we demonstrate the practical behaviour of the approximation scheme on concrete numerical examples.

Throughout this paper, $C$ will denote a generic strictly positive constant, independent of both the discretization and thickness parameters (respectively $h$ and $d$ ), that will take different values at different occurences (including when repeated in a single equation).

\section{Geometry and continuous formulation}

The geometry of the rod is described by a smooth function $\boldsymbol{\alpha}:[0, L] \rightarrow \mathbb{R}^{3}$, parametrized by arc-length $s$, which represents the line of centroids. We let $\mathcal{I} \stackrel{\text { def }}{=}$ $[0, L]$ and denote by $\boldsymbol{t}$ the unit tangent vector, so that $\boldsymbol{t}=\frac{d \boldsymbol{\alpha}}{d s}$ (see Figure 1). The linear elastic model we analyse, very similar to that used in [3], is a straightforward extension of the Timoshenko beam theory to a curved geometry, with bending, shear, torsion and membrane effects. Its variational formulation reads

$$
\int_{0}^{L} \tilde{\mathcal{J}}\left(\frac{d \boldsymbol{\theta}}{d s}\right) \cdot \frac{d \boldsymbol{\psi}}{d s} d s+\int_{0}^{L} \tilde{\mathcal{A}}\left[\frac{d \boldsymbol{u}}{d s}-\boldsymbol{\theta} \wedge \boldsymbol{t}\right] \cdot\left(\frac{d \boldsymbol{v}}{d s}-\boldsymbol{\psi} \wedge \boldsymbol{t}\right) d s=\int_{0}^{L} \boldsymbol{f} \cdot \boldsymbol{v} d s
$$

Here $\boldsymbol{u}$ and $\boldsymbol{\theta}$ respectively stand for the displacement and rotation vectors of a section of the rod, whereas $\boldsymbol{v}$ and $\boldsymbol{\psi}$ denote the corresponding trial functions. Vector $\boldsymbol{f}$ 


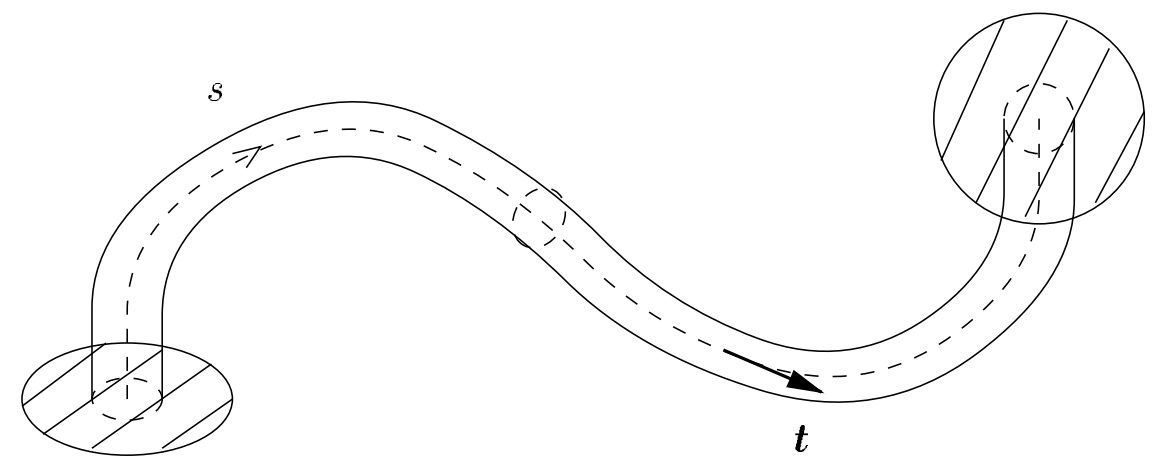

Figure 1: Rod with arbitrary three-dimensional geometry

represents the distributed load, while $\tilde{\mathcal{J}}$ and $\tilde{\mathcal{A}}$ are $s$-dependent linear operators. We assume that, in any local orthogonal coordinate system where the first coordinate is attached to $\boldsymbol{t}$, the matrix forms of $\tilde{\mathcal{J}}$ and $\tilde{\mathcal{A}}$ respectively reduce to $\operatorname{diag}(G J, E I, E I)$ and $\operatorname{diag}(E A, G A k, G A k)$, which means that the bending and shear stiffnesses are independent of the specific normal direction considered. Here $E, G, I, J, A$ and $k$ respectively denote Young's modulus, the shear modulus, the principal and polar moments of inertia, the cross-sectional area and the shear correction factor.

For very thin structures, it is well known that standard finite element procedures, when used in formulations such as (1), are subject to numerical locking, a phenomenon induced by the difference of magnitude between the coefficients in front of the different terms [1]. The appropriate framework for analysing this difficulty is obtained by rescaling formulation (1), so as to identify a well-posed sequence of problems in the limit where the thickness becomes infinitely small. Calling $d$ a non-dimensional parameter characteristic of the thickness (say $d=\sqrt{\frac{I}{A L^{2}}}$ ), this is reached by dividing both sides of equation (1) by $d^{4}$. We rewrite the result in the following form

$$
\int_{0}^{L} \mathcal{J}\left(\frac{d \boldsymbol{\theta}}{d s}\right) \cdot \frac{d \boldsymbol{\psi}}{d s} d s+d^{-2} \int_{0}^{L} \mathcal{A}\left[\frac{d \boldsymbol{u}}{d s}-\boldsymbol{\theta} \wedge \boldsymbol{t}\right] \cdot\left(\frac{d \boldsymbol{v}}{d s}-\boldsymbol{\psi} \wedge \boldsymbol{t}\right) d s=\int_{0}^{L} \boldsymbol{p} \cdot \boldsymbol{v} d s
$$

with $\mathcal{J}=\operatorname{diag}\left(j_{t}, j_{n}, j_{n}\right)$ and $\mathcal{A}=\operatorname{diag}\left(a_{t}, a_{n}, a_{n}\right)$ in a coordinate system as described before. These coefficients of $\mathcal{A}$ and $\mathcal{J}$ are taken as constants in the problem sequence in consideration, and so is $\boldsymbol{p}$, so that only $d$ varies in this equation.

RR n 2733 
This means that we study the problem sequence where the global size of a section varies while its shape, as well as the geometry of the line of centroids and the material properties remain unchanged, with a loading proportional to $d^{4}$. The values of practical interest for $d$ are obviously bounded, so we will assume $\left.d \in] 0, d_{0}\right]$ in the sequel.

We will also assume in our analysis that the rod we consider is not a simple straight beam. This problem would feature significant technical differences that could be dealt with separately, and the finite element scheme we propose is obviously well-fitted in this case too. However, this question has been specifically and extensively treated elsewhere (see [1] and the references therein). Furthermore, without loss of generality we concentrate on the case where the rod is clamped at both ends. The appropriate functional space in which solutions are to be sought appears to be $V \stackrel{\text { def }}{=}\left[H_{0}^{1}(\mathcal{I})\right]^{6}$ so that we can now state our problem in the more precise manner:

$\left(\mathcal{P}_{d}\right)$ : Find $(\boldsymbol{u}, \boldsymbol{\theta})$ in $V$ such that (2) holds for any $(\boldsymbol{v}, \boldsymbol{\psi})$ in $V$.

Proposition 2.1 Problem $\left(\mathcal{P}_{d}\right)$ has a unique solution $(\boldsymbol{u}, \boldsymbol{\theta})$ in $V$. Moreover, this solution satisfies the a priori estimate:

$$
\|\boldsymbol{u}, \boldsymbol{\theta}\|_{1} \leq C\|\boldsymbol{p}\|_{0}
$$

where $C$ is independent of $d$.

Proof: Let

$a_{d}(\boldsymbol{u}, \boldsymbol{\theta} ; \boldsymbol{v}, \boldsymbol{\psi}) \stackrel{\text { def }}{=} \int_{0}^{L} \mathcal{J}\left(\frac{d \boldsymbol{\theta}}{d s}\right) \cdot \frac{d \boldsymbol{\psi}}{d s} d s+d^{-2} \int_{0}^{L} \mathcal{A}\left[\frac{d \boldsymbol{u}}{d s}-\boldsymbol{\theta} \wedge \boldsymbol{t}\right] \cdot\left(\frac{d \boldsymbol{v}}{d s}-\boldsymbol{\psi} \wedge \boldsymbol{t}\right) d s$

The bilinear and continuous character of $a_{d}$ is evident. Moreover

$$
\begin{aligned}
a_{d}(\boldsymbol{u}, \boldsymbol{\theta} ; \boldsymbol{u}, \boldsymbol{\theta}) \geq & \min \left(j_{t}, j_{n}\right) \int_{0}^{L}\left\|\frac{d \boldsymbol{\theta}}{d s}\right\|^{2} d s \\
& +\min \left(a_{t}, a_{n}\right) d^{-2} \int_{0}^{L}\left\|\frac{d \boldsymbol{u}}{d s}-\boldsymbol{\theta} \wedge \boldsymbol{t}\right\|^{2} d s \\
\geq & C\left\{\left\|\frac{d \boldsymbol{\theta}}{d s}\right\|_{0}^{2}+\left\|\frac{d \boldsymbol{u}}{d s}-\boldsymbol{\theta} \wedge \boldsymbol{t}\right\|_{0}^{2}\right\} \\
\geq & C\|\boldsymbol{u}, \boldsymbol{\theta}\|_{1}^{2}
\end{aligned}
$$


by the Poincare inequality, so that uniform ellipticity with respect to $d$ holds. Existence, uniqueness and estimate (3) immediately follow.

We now introduce a mixed formulation equivalent to $\left(\mathcal{P}_{d}\right)$. We set

$$
\begin{gathered}
B(\boldsymbol{u}, \boldsymbol{\theta}, \boldsymbol{\lambda} ; \boldsymbol{v}, \boldsymbol{\psi}, \boldsymbol{\mu}) \stackrel{\text { def }}{=} \int_{0}^{L} \mathcal{J}\left(\frac{d \boldsymbol{\theta}}{d s}\right) \cdot \frac{d \boldsymbol{\psi}}{d s} d s+\int_{0}^{L} \boldsymbol{\lambda} \cdot\left(\frac{d \boldsymbol{v}}{d s}-\boldsymbol{\psi} \wedge \boldsymbol{t}\right) d s \\
+\int_{0}^{L} \boldsymbol{\mu} \cdot\left(\frac{d \boldsymbol{u}}{d s}-\boldsymbol{\theta} \wedge \boldsymbol{t}\right) d s-d^{2} \int_{0}^{L} \mathcal{A}^{-1}(\boldsymbol{\lambda}) \cdot \boldsymbol{\mu} d s \\
F(\boldsymbol{v}) \stackrel{\text { def }}{=} \int_{0}^{L} \boldsymbol{p} \cdot \boldsymbol{v} d s
\end{gathered}
$$

We define $Q \stackrel{\text { def }}{=}\left[L^{2}(\mathcal{I})\right]^{3}$ and consider the new problem:

$\left(\mathcal{P}_{d}^{\prime}\right):$ Find $(\boldsymbol{u}, \boldsymbol{\theta}, \boldsymbol{\lambda})$ in $V \times Q$ such that

$$
B(\boldsymbol{u}, \boldsymbol{\theta}, \boldsymbol{\lambda} ; \boldsymbol{v}, \boldsymbol{\psi}, \boldsymbol{\mu})=F(\boldsymbol{v}) \quad \forall(\boldsymbol{v}, \boldsymbol{\psi}, \boldsymbol{\mu}) \in V \times Q
$$

To see that $\left(\mathcal{P}_{d}^{\prime}\right)$ and $\left(\mathcal{P}_{d}\right)$ are equivalent is straightforward: taking $(\boldsymbol{v}, \boldsymbol{\psi})=0$ in (4) yields

$$
\boldsymbol{\lambda}=d^{-2} \mathcal{A}\left(\frac{d \boldsymbol{u}}{d s}-\boldsymbol{\theta} \wedge \boldsymbol{t}\right)
$$

then substituting into (4) and taking $\boldsymbol{\mu}=0$, one gets (2). Yet, in order to derive a bound independent of $d$ for $\boldsymbol{\lambda}$, we need to devote more care to $\left(\mathcal{P}_{d}^{\prime}\right)$ specifically.

Proposition 2.2 Problem $\left(\mathcal{P}_{d}^{\prime}\right)$ has a unique solution $(\boldsymbol{u}, \boldsymbol{\theta}, \boldsymbol{\lambda})$. This solution satisfies

$$
\|\boldsymbol{u}, \boldsymbol{\theta}\|_{1}+\|\boldsymbol{\lambda}\|_{0} \leq C\|\boldsymbol{p}\|_{0}
$$

Proof: Problem $\left(\mathcal{P}_{d}^{\prime}\right)$ is a standard mixed formulation in the sense of [6]. Defining

$$
\begin{gathered}
a(\boldsymbol{u}, \boldsymbol{\theta} ; \boldsymbol{v}, \boldsymbol{\psi}) \stackrel{\text { def }}{=} \int_{0}^{L} \mathcal{J}\left(\frac{d \boldsymbol{\theta}}{d s}\right) \cdot \frac{d \boldsymbol{\psi}}{d s} d s \\
b(\boldsymbol{v}, \boldsymbol{\psi} ; \boldsymbol{\lambda}) \stackrel{\text { def }}{=} \int_{0}^{L} \boldsymbol{\lambda} \cdot\left(\frac{d \boldsymbol{v}}{d s}-\boldsymbol{\psi} \wedge \boldsymbol{t}\right) d s \\
V_{0} \stackrel{\text { def }}{=}\{(\boldsymbol{v}, \boldsymbol{\psi}) \in V ; b(\boldsymbol{v}, \boldsymbol{\psi} ; \boldsymbol{\mu})=0, \forall \boldsymbol{\mu} \in Q\}
\end{gathered}
$$

RR $n^{\circ} 2733$ 
we know that the result we want to establish will hold if and only if two conditions are satisfied (see Theorem II.1.2 and Remark II.1.13 in [6]):

i) $a$ must be coercive on $V_{0}$.

ii) $b$ must satisfy the inf-sup condition:

$$
\inf _{\boldsymbol{\mu} \in Q} \sup _{(\boldsymbol{v}, \boldsymbol{\psi}) \in V} \frac{b(\boldsymbol{v}, \boldsymbol{\psi} ; \boldsymbol{\mu})}{\|\boldsymbol{\mu}\|_{0}\|\boldsymbol{v}, \boldsymbol{\psi}\|_{1}} \geq C>0
$$

To check condition i), we just have to note that, for any $(\boldsymbol{v}, \boldsymbol{\psi}) \in V_{0}$

$$
a(\boldsymbol{v}, \boldsymbol{\psi} ; \boldsymbol{v}, \boldsymbol{\psi})=a_{d}(\boldsymbol{v}, \boldsymbol{\psi} ; \boldsymbol{v}, \boldsymbol{\psi})
$$

so that the coercivity of $a$ on $V_{0}$ follows from that of $a_{d}$ on the whole of $V$. For condition ii) we need a preparatory result.

Lemma 2.1 There exists a linear mapping $\phi: \mathbb{R}^{3} \rightarrow \mathcal{C}^{1}\left(\mathcal{I}, \mathbb{R}^{3}\right)$ such that, for any $\boldsymbol{x} \in \mathbb{R}^{3}$ :

$$
\begin{gathered}
\phi[\boldsymbol{x}](0)=\boldsymbol{\phi}[\boldsymbol{x}](L)=\mathbf{0} \\
\int_{0}^{L} \phi[\boldsymbol{x}] \wedge \boldsymbol{t} d s=\boldsymbol{x} \\
\|\boldsymbol{\phi}[\boldsymbol{x}]\|_{\mathcal{C}^{1}} \leq C\|\boldsymbol{x}\|
\end{gathered}
$$

Proof: $\quad$ Let $\Omega \stackrel{\text { def }}{=}\left\{\boldsymbol{\omega} \in \mathcal{C}^{1}\left(\mathcal{I}, \mathbb{R}^{3}\right) ; \boldsymbol{\omega}(0)=\boldsymbol{\omega}(L)=\mathbf{0}\right\}$ and define the operator $\mathcal{F}: \Omega \rightarrow \mathbb{R}^{3}$ by

$$
\mathcal{F}(\boldsymbol{\omega}) \stackrel{\text { def }}{=} \int_{0}^{L} \boldsymbol{\omega} \wedge \boldsymbol{t} d s
$$

$\Omega$ is a vector space and $\mathcal{F}$ a linear mapping so that its range is a subspace of $\mathbb{R}^{3}$. Then let $\boldsymbol{x}_{0}$ be any vector of $\mathbb{R}^{3}$ orthogonal to this subspace, i.e.

$$
\boldsymbol{x}_{0} \cdot \mathcal{F}(\boldsymbol{\omega})=0 \quad \forall \boldsymbol{\omega} \in \Omega
$$

Define $\beta(s) \stackrel{\text { def }}{=} s(L-s)$ and choose in (11) $\boldsymbol{\omega}=\beta \boldsymbol{t} \wedge \boldsymbol{x}_{0}$ then we infer

$$
\boldsymbol{x}_{0} \cdot \int_{0}^{L} \boldsymbol{\omega} \wedge \boldsymbol{t} d s=\int_{0}^{L} \beta\left\|\boldsymbol{t} \wedge \boldsymbol{x}_{0}\right\|^{2} d s=0
$$


so that $\boldsymbol{t} \wedge \boldsymbol{x}_{0}$ must be identically zero, which implies $\boldsymbol{x}_{0}=0$ since the rod is not a straight beam. Therefore $\mathcal{F}$ is surjective. Now, to build the mapping $\phi$ it suffices to arbitrarily choose 3 elements of $\Omega$, the image of which are the 3 base vectors of $\mathbb{R}^{3}$.

Proof of Proposition 2.2 (continued): To prove that condition ii) is satisfied, we will show that, for any $\boldsymbol{\lambda} \in Q$, we can build a displacement field $(\boldsymbol{v}, \boldsymbol{\psi}) \in V$ such that

$$
\begin{gathered}
\frac{d \boldsymbol{v}}{d s}-\boldsymbol{\psi} \wedge \boldsymbol{t}=\boldsymbol{\lambda} \\
\|\boldsymbol{v}, \boldsymbol{\psi}\|_{1} \leq C\|\boldsymbol{\lambda}\|_{0}
\end{gathered}
$$

We start by taking

$$
\begin{gathered}
\boldsymbol{v}^{(1)} \stackrel{\text { def }}{=} \int_{0}^{s} \boldsymbol{\lambda} d s \\
\boldsymbol{\psi}^{(1)} \stackrel{\text { def }}{=} \mathbf{0}
\end{gathered}
$$

This displacement field satisfies the first requirement (12), and its norm is clearly bounded:

$$
\left\|\boldsymbol{v}^{(1)}, \boldsymbol{\psi}^{(1)}\right\|_{1} \leq C\|\boldsymbol{\lambda}\|_{0}
$$

but $\boldsymbol{v}^{(1)}(L) \neq 0$ in general, so that $\boldsymbol{v}^{(1)}$ fails to satisfy the proper boundary conditions. However, letting $\boldsymbol{x} \stackrel{\text { def }}{=}-\boldsymbol{v}^{(1)}(L)$, we have

$$
\|\boldsymbol{x}\| \leq C\left\|\boldsymbol{v}^{(1)}\right\|_{1} \leq C\|\boldsymbol{\lambda}\|_{0}
$$

and we now apply Lemma 2.1 to define:

$$
\begin{gathered}
\boldsymbol{\psi}^{(2)} \stackrel{\text { def }}{=} \phi[\boldsymbol{x}] \\
\boldsymbol{v}^{(2)} \stackrel{\text { def }}{=} \int_{0}^{s} \phi[\boldsymbol{x}] \wedge \boldsymbol{t} d s
\end{gathered}
$$

so that

$$
\frac{d \boldsymbol{v}^{(2)}}{d s}-\boldsymbol{\psi}^{(2)} \wedge \boldsymbol{t}=\mathbf{0}
$$

From (9) we have:

$$
\boldsymbol{v}^{(2)}(L)=\boldsymbol{x}=-\boldsymbol{v}^{(1)}(L)
$$

RR n 2733 
Moreover (10) and (15) imply

$$
\left\|\boldsymbol{v}^{(2)}, \boldsymbol{\psi}^{(2)}\right\|_{1} \leq C\|\boldsymbol{\lambda}\|_{0}
$$

Finally, the choice

$$
\left.\begin{array}{l}
\boldsymbol{v} \stackrel{\text { def }}{=} \boldsymbol{v}^{(1)}+\boldsymbol{v}^{(2)} \\
\boldsymbol{\psi} \stackrel{\text { def }}{=} \boldsymbol{\psi}^{(1)}+\boldsymbol{\psi}^{(2)}
\end{array}\right\}
$$

satisfies conditions (12) and (13) as well as the rightboundary conditions.

Remark 2.1: Had we been considering a structure clamped at one end only, the proof at the inf-sup stage would have been much simpler, with no correction necessary on $\left(\boldsymbol{v}^{(1)}, \boldsymbol{\psi}^{(1)}\right)$. This same remark will hold for the discrete inf-sup condition in the next section. Thus, we can see that the boundary conditions we have chosen to consider are in fact the most severe possible case.

We end this section with a regularity result for a problem slightly more general than $\left(\mathcal{P}_{d}^{\prime}\right)$. Consider

$\left(\tilde{\mathcal{P}}_{d}^{\prime}\right)$ : Find $(\tilde{\boldsymbol{u}}, \tilde{\boldsymbol{\theta}}, \tilde{\boldsymbol{\lambda}})$ in $V \times Q$ such that

$$
B(\tilde{\boldsymbol{u}}, \tilde{\boldsymbol{\theta}}, \tilde{\boldsymbol{\lambda}} ; \boldsymbol{v}, \boldsymbol{\psi}, \boldsymbol{\mu})=\int_{0}^{L} \boldsymbol{p} \cdot \boldsymbol{v} d s+\int_{0}^{L} \boldsymbol{q} \cdot \boldsymbol{\psi} d s \quad \forall(\boldsymbol{v}, \boldsymbol{\psi}, \boldsymbol{\mu}) \in V \times Q
$$

This extension with the rotation appearing in the right hand side will be needed to derive $L^{2}$ error estimates by a duality argument.

Proposition 2.3 Problem $\left(\tilde{\mathcal{P}}_{d}^{\prime}\right)$ has a unique solution $(\tilde{\boldsymbol{u}}, \tilde{\boldsymbol{\theta}}, \tilde{\boldsymbol{\lambda}})$ in $V \times Q$. This solution is also in $\left[H^{2}(\mathcal{I})\right]^{6} \times\left[H^{1}(\mathcal{I})\right]^{3}$ and satisfies

$$
\|\tilde{\boldsymbol{u}}\|_{2}+\|\tilde{\boldsymbol{\theta}}\|_{2}+\|\tilde{\boldsymbol{\lambda}}\|_{1} \leq C\left\{\|\boldsymbol{p}\|_{0}+\|\boldsymbol{q}\|_{0}\right\}
$$

Proof: The existence and uniqueness of a solution for $\left(\tilde{\mathcal{P}}_{d}^{\prime}\right)$ stems from the exact same argument as for $\left(\mathcal{P}_{d}^{\prime}\right)$, which also implies the following a priori bound:

$$
\|\tilde{\boldsymbol{u}}\|_{1}+\|\tilde{\boldsymbol{\theta}}\|_{1}+\|\tilde{\boldsymbol{\lambda}}\|_{0} \leq C\left\{\|\boldsymbol{p}\|_{0}+\|\boldsymbol{q}\|_{0}\right\}
$$


To prove one additional level of regularity, we write the differential equations satisfied by the solution in the distribution sense:

$$
\begin{gathered}
-\frac{d \tilde{\boldsymbol{\lambda}}}{d s}=\boldsymbol{p} \\
-\frac{d}{d s}\left[\mathcal{J}\left(\frac{d \tilde{\boldsymbol{\theta}}}{d s}\right)\right]-\boldsymbol{t} \wedge \tilde{\boldsymbol{\lambda}}=\boldsymbol{q} \\
\frac{d \tilde{\boldsymbol{u}}}{d s}-\tilde{\boldsymbol{\theta}} \wedge \boldsymbol{t}-d^{2} \mathcal{A}^{-1}(\tilde{\boldsymbol{\lambda}})=\mathbf{0}
\end{gathered}
$$

Assume that $\boldsymbol{p}$ and $\boldsymbol{q}$ are in $\left[L^{2}(\mathcal{I})\right]^{3}$. From (21) and (22) we infer that $\tilde{\boldsymbol{\lambda}}$ is in $\left[H^{1}(\mathcal{I})\right]^{3}$ and

$$
\|\tilde{\boldsymbol{\lambda}}\|_{1} \leq C\left\{\|\boldsymbol{p}\|_{0}+\|\boldsymbol{q}\|_{0}\right\}
$$

Let us now write an explicit expression for $\mathcal{J}$ and $\mathcal{J}^{-1}$ :

$$
\left.\begin{array}{l}
\mathcal{J}(\boldsymbol{x})=\left(j_{t}-j_{n}\right)(\boldsymbol{x} \cdot \boldsymbol{t}) \boldsymbol{t}+j_{n} \boldsymbol{x} \\
\mathcal{J}^{-1}(\boldsymbol{x})=\left(j_{t}^{-1}-j_{n}^{-1}\right)(\boldsymbol{x} \cdot \boldsymbol{t}) \boldsymbol{t}+j_{n}^{-1} \boldsymbol{x}
\end{array}\right\}
$$

and similar expressions hold for $\mathcal{A}$ and $\mathcal{A}^{-1}$. Observe that all these operators preserve the Sobolev spaces on which they act. Thus, (21) and (23) imply that $\tilde{\boldsymbol{\theta}}$ is in $\left[H^{2}(\mathcal{I})\right]^{3}$ with

$$
\|\tilde{\boldsymbol{\theta}}\|_{2} \leq C\left\{\|\boldsymbol{p}\|_{0}+\|\boldsymbol{q}\|_{0}\right\}
$$

Similarly (21) and (24) entail $\tilde{\boldsymbol{u}} \in\left[H^{2}(\mathcal{I})\right]^{3}$ and

$$
\|\tilde{\boldsymbol{u}}\|_{2} \leq C\left\{\|\boldsymbol{p}\|_{0}+\|\boldsymbol{q}\|_{0}\right\}
$$

\section{Approximation by straight beam elements}

In [3] Arunakirinathar and Reddy investigate the direct approximation of problem $\left(\mathcal{P}_{d}^{\prime}\right)$ by a regular mixed finite element method. Our approach is completely different

RR n 2733 
and generalizes that of Kikuchi in [9] (see also [8, 5, 11]). First, we approximate the structure geometrically by an assembly of straight beams, connected by rigid junctions. Then, to each straight beam in this set we substitute a simple beam finite element to compute the actual approximation.

The geometric approximation is characterized by a set of points $\left(M_{i}\right)_{i=0}^{N}$ on the line of centroids, corresponding to arc-lengths $\left(s_{i}\right)_{i=0}^{N}$, such that $s_{0}=0, s_{N}=L$, and for any $i(0 \leq i \leq N-1), 0<s_{i+1}-s_{i} \leq h$. The assembly of beams is obtained by joining two adjacent points by a straight line. We denote by $\bar{s}$ the arc-length along this piecewise-straight curve, $\left(\bar{s}_{i}\right)_{i=0}^{N}$ the corresponding values at points $M_{i}$, $\bar{L}$ the total length, $\overline{\mathcal{I}}$ the interval $[0, \bar{L}]$ and $\overline{\boldsymbol{t}}$ the unit tangent vector wherever it is uniquely defined (i.e. everywhere except at points $M_{i}$ ).

We now describe the finite element procedure. Define $\bar{V}_{h}$ as the space of functions of $\left[H_{0}^{1}(\overline{\mathcal{I}})\right]^{6}$ that are piecewise-linear in each subinterval $\left[\bar{s}_{i}, \bar{s}_{i+1}\right]$. We consider the following problem:

$\left(\mathcal{P}_{d, h}\right):$ Find $\left(\boldsymbol{u}_{h}, \boldsymbol{\theta}_{h}\right)$ in $\bar{V}_{h}$ such that

$$
\begin{gathered}
\int_{0}^{\bar{L}} \overline{\mathcal{J}}\left(\frac{d \boldsymbol{\theta}_{h}}{d \bar{s}}\right) \cdot \frac{d \boldsymbol{\psi}_{h}}{d \bar{s}} d \bar{s} \\
+d^{-2} \int_{0}^{\bar{L}} \Pi_{h}\left(\overline{\mathcal{A}}\left[\frac{d \boldsymbol{u}_{h}}{d \bar{s}}-\boldsymbol{\theta}_{h} \wedge \overline{\boldsymbol{t}}\right]\right) \cdot\left(\frac{d \boldsymbol{v}_{h}}{d \bar{s}}-\boldsymbol{\psi}_{h} \wedge \overline{\boldsymbol{t}}\right) d \bar{s}=\int_{0}^{\bar{L}} \boldsymbol{p} \cdot \boldsymbol{v}_{h} d \bar{s} \\
\forall\left(\boldsymbol{v}_{h}, \boldsymbol{\psi}_{h}\right) \in \bar{V}_{h}
\end{gathered}
$$

where operators $\overline{\mathcal{J}}$ and $\overline{\mathcal{A}}$ have the same diagonal form as $\mathcal{J}$ and $\mathcal{A}$, but in a local coordinate system attached to $\overline{\boldsymbol{t}}$ instead of $\boldsymbol{t}$. $\Pi_{h}$ denotes the $L^{2}$-projector onto piecewise-constants. To each function defined on $\mathcal{I}$, we associate one defined on $\overline{\mathcal{I}}$ using the piecewise-linear change of variables in which each $\bar{s}_{i}$ corresponds to $s_{i}$. Conversely, we will use the inverse change of variables to derive functions defined on $\mathcal{I}$ from functions defined on $\overline{\mathcal{I}}$. In both cases, we will not distinguish between the two functions in the notation. However the context will make it clear whether the one or the other is to be understood, like for $\boldsymbol{p}$ in the right hand side of (29).

Remark 3.1: The finite elements we employ are simple 3D extensions of linear Timoshenko beam elements, including torsion and membrane deformations in addition to shear and bending, with one-point selective reduced integration on the membrane/shear term. This reduced integration strategy is natural, since for the 
Timoshenko beam model a similar modification of the shear term is proved to overcome numerical locking [1].

Like in the continuous case, we proceed to write an equivalent mixed problem. Define the auxiliary unknown $\boldsymbol{\lambda}_{h}$, related to $\left(\boldsymbol{u}_{h}, \boldsymbol{\theta}_{h}\right)$ by

$$
\boldsymbol{\lambda}_{h} \stackrel{d e f}{=} d^{-2} \Pi_{h}\left\{\overline{\mathcal{A}}\left[\frac{d \boldsymbol{u}_{h}}{d \bar{s}}-\boldsymbol{\theta}_{h} \wedge \overline{\boldsymbol{t}}\right]\right\}
$$

Note that there is no difficulty involved in the interaction between $\Pi_{h}$ and $\overline{\mathcal{A}}$ since $\overline{\boldsymbol{t}}$ is a constant within each element, so that the two operators commute. With $Q_{h}$ defined as the space of piecewise-constants over $\overline{\mathcal{I}}$ valued in $\mathbb{R}^{3}$, the discrete mixed problem is

$\left(\mathcal{P}_{d, h}^{\prime}\right):$ Find $\left(\boldsymbol{u}_{h}, \boldsymbol{\theta}_{h}, \boldsymbol{\lambda}_{h}\right)$ in $\bar{V}_{h} \times \bar{Q}_{h}$ such that

$$
B_{h}\left(\boldsymbol{u}_{h}, \boldsymbol{\theta}_{h}, \boldsymbol{\lambda}_{h} ; \boldsymbol{v}_{h}, \boldsymbol{\psi}_{h}, \boldsymbol{\mu}_{h}\right)=F_{h}\left(\boldsymbol{v}_{h}\right) \quad \forall\left(\boldsymbol{v}_{h}, \boldsymbol{\psi}_{h}, \boldsymbol{\mu}_{h}\right) \in \bar{V}_{h} \times \bar{Q}_{h}
$$

where

$$
\begin{gathered}
B_{h}\left(\boldsymbol{u}_{h}, \boldsymbol{\theta}_{h}, \boldsymbol{\lambda}_{h} ; \boldsymbol{v}_{h}, \boldsymbol{\psi}_{h}, \boldsymbol{\mu}_{h}\right) \stackrel{d e f}{=} \int_{0}^{\bar{L}} \overline{\mathcal{J}}\left(\frac{d \boldsymbol{\theta}_{h}}{d \bar{s}}\right) \cdot \frac{d \boldsymbol{\psi}_{h}}{d \bar{s}} d \bar{s}+\int_{0}^{\bar{L}} \boldsymbol{\lambda}_{h} \cdot\left(\frac{d \boldsymbol{v}_{h}}{d \bar{s}}-\boldsymbol{\psi}_{h} \wedge \overline{\boldsymbol{t}}\right) d \bar{s} \\
+\int_{0}^{\bar{L}} \boldsymbol{\mu}_{h} \cdot\left(\frac{d \boldsymbol{u}_{h}}{d \bar{s}}-\boldsymbol{\theta}_{h} \wedge \overline{\boldsymbol{t}}\right) d \bar{s}-d^{2} \int_{0}^{\bar{L}} \overline{\mathcal{A}}^{-1}\left(\boldsymbol{\lambda}_{h}\right) \cdot \boldsymbol{\mu}_{h} d \bar{s} \\
F_{h}\left(\boldsymbol{v}_{h}\right) \stackrel{d e f}{=} \int_{0}^{\bar{L}} \boldsymbol{p} \cdot \boldsymbol{v}_{h} d \bar{s}
\end{gathered}
$$

Let us call $V_{h}$ the space of functions in $\left[H_{0}^{1}(\mathcal{I})\right]^{6}$ that are piecewise-linear within each $\left[s_{i}, s_{i+1}\right]$, and $Q_{h}$ the space of piecewise-constant functions valued in $\mathbb{R}^{3}$. By the canonical change of variables between $\mathcal{I}$ and $\overline{\mathcal{I}}$ that we described, one can construct an element of $V_{h}$ from one of $\bar{V}_{h}$ and vice-versa, and the same holds for $Q_{h}$ and $\bar{Q}_{h}$. Therefore $\left(\mathcal{P}_{d, h}\right)$ can be seen as a candidate for approximating $\left(\mathcal{P}_{d}\right)$, or $\left(\mathcal{P}_{d, h}^{\prime}\right)$ for $\left(\mathcal{P}_{d}^{\prime}\right)$. In the remainder of this study, we will investigate the convergence of the mixed scheme, keeping in mind that it implies the convergence of the diplacement-based formulation.

We start with results of geometric nature that will be needed to relate functions defined on $\mathcal{I}$ and $\overline{\mathcal{I}}$. We define

$$
s_{i+\frac{1}{2}} \stackrel{\text { def }}{=} \frac{1}{2}\left(s_{i}+s_{i+1}\right)
$$

RR n'2733 
We will use the standard notation $O$ and $\mathbf{0}$ for respectively scalar and vector infinitesimals which do not depend on parameter $d$.

Lemma 3.1 Within each subinterval $\left[s_{i}, s_{i+1}\right]$, the following properties hold with bounding constants valid over the whole of $\mathcal{I}$.

$$
\begin{aligned}
& \left.\begin{array}{l}
1 \leq \frac{d s}{d \bar{s}} \leq 1+C h^{2} \\
1-C h^{2} \leq \frac{d \bar{s}}{d s} \leq 1
\end{array}\right\} \\
& \overline{\boldsymbol{t}}=\boldsymbol{t}\left(s_{i+\frac{1}{2}}\right)+\mathbf{0}\left(h^{2}\right) \\
& \boldsymbol{t}(s)=\boldsymbol{t}\left(s_{i+\frac{1}{2}}\right)+\left(s-s_{i+\frac{1}{2}}\right) \frac{d^{2} \boldsymbol{\alpha}}{d s^{2}}\left(s_{i+\frac{1}{2}}\right)+\mathbf{0}\left(\left|s-s_{i+\frac{1}{2}}\right|^{2}\right)
\end{aligned}
$$

Proof: The Taylor expansion of the mapping $\boldsymbol{\alpha}$ at $s_{i+\frac{1}{2}}$ reads:

$$
\boldsymbol{\alpha}(s)=\boldsymbol{\alpha}\left(s_{i+\frac{1}{2}}\right)+\left(s-s_{i+\frac{1}{2}}\right) \boldsymbol{t}\left(s_{i+\frac{1}{2}}\right)+\frac{\left(s-s_{i+\frac{1}{2}}\right)^{2}}{2} \frac{d^{2} \boldsymbol{\alpha}}{d s^{2}}\left(s_{i+\frac{1}{2}}\right)+\mathbf{0}\left(\left|s-s_{i+\frac{1}{2}}\right|^{3}\right)
$$

Writing (35) for $s=s_{i}$ and $s=s_{i+1}$ and subtracting yields:

$$
\frac{\boldsymbol{\alpha}\left(s_{i+1}\right)-\boldsymbol{\alpha}\left(s_{i}\right)}{s_{i+1}-s_{i}}=\boldsymbol{t}\left(s_{i+\frac{1}{2}}\right)+\boldsymbol{0}\left(h^{2}\right)
$$

Therefore

$$
\frac{d \bar{s}}{d s}=\left\|\frac{\boldsymbol{\alpha}\left(s_{i+1}\right)-\boldsymbol{\alpha}\left(s_{i}\right)}{s_{i+1}-s_{i}}\right\|=1+O\left(h^{2}\right)
$$

and since by an obvious geometric reasoning

$$
\frac{d \bar{s}}{d s} \leq 1
$$

(32) is proved. Then

$$
\overline{\boldsymbol{t}}=\frac{\boldsymbol{\alpha}\left(s_{i+1}\right)-\boldsymbol{\alpha}\left(s_{i}\right)}{\bar{s}_{i+1}-\bar{s}_{i}}=\frac{\boldsymbol{\alpha}\left(s_{i+1}\right)-\boldsymbol{\alpha}\left(s_{i}\right)}{s_{i+1}-s_{i}} \frac{d s}{d \bar{s}}
$$

so that combining (32) and (36) we obtain (33). Finally (34) is simply the Taylor expansion of $\boldsymbol{t}$ at $s_{i+\frac{1}{2}}$.

We now establish the stability of the mixed approximation scheme. 
Lemma 3.2 For $h$ small enough, $B_{h}$ provides a stable approximation procedure, i.e. for any $\left(\boldsymbol{u}_{h}, \boldsymbol{\theta}_{h}, \boldsymbol{\lambda}_{h}\right) \in V_{h} \times Q_{h}$, there exists $\left(\boldsymbol{v}_{h}, \boldsymbol{\psi}_{h}, \boldsymbol{\mu}_{h}\right) \in V_{h} \times Q_{h}$ such that

$$
\begin{gathered}
\left\|\boldsymbol{v}_{h}, \boldsymbol{\psi}_{h}\right\|_{1}+\left\|\boldsymbol{\mu}_{h}\right\|_{0} \leq\left\|\boldsymbol{u}_{h}, \boldsymbol{\theta}_{h}\right\|_{1}+\left\|\boldsymbol{\lambda}_{h}\right\|_{0} \\
B_{h}\left(\boldsymbol{u}_{h}, \boldsymbol{\theta}_{h}, \boldsymbol{\lambda}_{h} ; \boldsymbol{v}_{h}, \boldsymbol{\psi}_{h}, \boldsymbol{\mu}_{h}\right) \geq C\left\{\left\|\boldsymbol{u}_{h}, \boldsymbol{\theta}_{h}\right\|_{1}+\left\|\boldsymbol{\lambda}_{h}\right\|_{0}\right\}^{2}
\end{gathered}
$$

Proof: In 3 steps.

i) Stability in $\boldsymbol{\theta}_{h}$. Take $\left(\boldsymbol{v}_{h}, \boldsymbol{\psi}_{h}, \boldsymbol{\mu}_{h}\right)=\left(\boldsymbol{u}_{h}, \boldsymbol{\theta}_{h},-\boldsymbol{\lambda}_{h}\right)$. Then

$$
\begin{aligned}
&\left\|\boldsymbol{v}_{h}, \boldsymbol{\psi}_{h}\right\|_{1}+\left\|\boldsymbol{\mu}_{h}\right\|_{0}=\left\|\boldsymbol{u}_{h}, \boldsymbol{\theta}_{h}\right\|_{1}+\left\|\boldsymbol{\lambda}_{h}\right\|_{0} \\
& B_{h}\left(\boldsymbol{u}_{h}, \boldsymbol{\theta}_{h}, \boldsymbol{\lambda}_{h} ; \boldsymbol{v}_{h}, \boldsymbol{\psi}_{h}, \boldsymbol{\mu}_{h}\right)=\int_{0}^{\bar{L}} \overline{\mathcal{J}}\left(\frac{d \boldsymbol{\theta}_{h}}{d \bar{s}}\right) \cdot \frac{d \boldsymbol{\theta}_{h}}{d \bar{s}} d \bar{s}+d^{2} \int_{0}^{\bar{L}} \overline{\mathcal{A}}^{-1}\left(\boldsymbol{\lambda}_{h}\right) \cdot \boldsymbol{\lambda}_{h} d \bar{s} \\
& \geq C \int_{0}^{\bar{L}}\left\|\frac{d \boldsymbol{\theta}_{h}}{d \bar{s}}\right\|^{2} d \bar{s}=C \int_{0}^{L}\left\|\frac{d \boldsymbol{\theta}_{h}}{d s}\right\|^{2} \frac{d s}{d \bar{s}} d s \\
& \geq C\left|\boldsymbol{\theta}_{h}\right|_{1}^{2} \geq C\left\|\boldsymbol{\theta}_{h}\right\|_{1}^{2}
\end{aligned}
$$

by (32) and the Poincaré inequality.

ii) Stability in $\boldsymbol{\lambda}_{h}$ (discrete inf-sup condition). The strategy here will follow the same main lines as in the proof of the continuous inf-sup condition, except that an equality like (12) will hold approximately only. Define:

$$
b_{h}\left(\boldsymbol{v}_{h}, \boldsymbol{\psi}_{h} ; \boldsymbol{\mu}_{h}\right) \stackrel{\text { def }}{=} \int_{0}^{\bar{L}} \boldsymbol{\mu}_{h} \cdot\left(\frac{d \boldsymbol{v}_{h}}{d \bar{s}}-\boldsymbol{\psi}_{h} \wedge \overline{\boldsymbol{t}}\right) d \bar{s}
$$

We start by choosing:

$$
\boldsymbol{v}_{h}^{(1)}(s) \stackrel{\text { def }}{=} \int_{0}^{s} \boldsymbol{\lambda}_{h} d s \quad \boldsymbol{\psi}_{h}^{(1)} \stackrel{\text { def }}{=} \mathbf{0} \quad \boldsymbol{\mu}_{h}^{(1)} \stackrel{\text { def }}{=} \mathbf{0}
$$

Note that with $\boldsymbol{\lambda}_{h}$ in $Q_{h}, \boldsymbol{v}_{h}^{(1)}$ so-defined is in the space of continuous piecewise-linear functions. Besides we have:

$$
\left\|\boldsymbol{v}_{h}^{(1)}\right\|_{1}^{2} \leq C\left|\boldsymbol{v}_{h}^{(1)}\right|_{1}^{2}=C\left\|\boldsymbol{\lambda}_{h}\right\|_{0}^{2}
$$

RR n'2733 
and

$$
\left.\begin{array}{rl}
B_{h}\left(\boldsymbol{u}_{h}, \boldsymbol{\theta}_{h}, \boldsymbol{\lambda}_{h} ; \boldsymbol{v}_{h}^{(1)}, \boldsymbol{\psi}_{h}^{(1)}, \boldsymbol{\mu}_{h}^{(1)}\right) & =b_{h}\left(\boldsymbol{v}_{h}^{(1)}, \mathbf{0} ; \boldsymbol{\lambda}_{h}\right)=\int_{0}^{\bar{L}} \boldsymbol{\lambda}_{h} \cdot \frac{d \boldsymbol{v}_{h}^{(1)}}{d \bar{s}} d \bar{s} \\
& =\int_{0}^{L} \boldsymbol{\lambda}_{h} \cdot \frac{d \boldsymbol{v}_{h}^{(1)}}{d s} d s=\left\|\boldsymbol{\lambda}_{h}\right\|_{0}^{2}
\end{array}\right\}
$$

However $\boldsymbol{v}_{h}^{(1)}(L)=\int_{0}^{L} \boldsymbol{\lambda}_{h} d s \neq \mathbf{0}$ in general, so that our choice does not fulfill the right boundary conditions. Nevertheless we record:

$$
\left\|\boldsymbol{v}_{h}^{(1)}(L)\right\| \leq C\left\|\boldsymbol{\lambda}_{h}\right\|_{0}
$$

Now set $\boldsymbol{x} \stackrel{\text { def }}{=}-\boldsymbol{v}_{h}^{(1)}(L)$. Denoting by $[\omega]_{\text {int }}$ the continuous piecewise-linear function that interpolates a continuous function $\omega$, we define:

$$
\begin{gathered}
\boldsymbol{\psi}_{h}^{(2)} \stackrel{\text { def }}{=}[\boldsymbol{\phi}[\boldsymbol{x}]]_{i n t} \\
\boldsymbol{v}_{h}^{(2)} \stackrel{\text { def }}{=}\left[\int_{0}^{s} \phi[\boldsymbol{x}] \wedge \boldsymbol{t} d s\right]_{i n t}
\end{gathered}
$$

where $\phi$ is the operator of Lemma 2.1. Then, using interpolation properties, (10) and (41) we have:

$$
\left\|\boldsymbol{\psi}_{h}^{(2)}\right\|_{1} \leq C\|\phi[\boldsymbol{x}]\|_{1} \leq C\|\boldsymbol{x}\| \leq C\left\|\boldsymbol{\lambda}_{h}\right\|_{0}
$$

and similarly

$$
\left\|\boldsymbol{v}_{h}^{(2)}\right\|_{1} \leq C\left|\boldsymbol{v}_{h}^{(2)}\right|_{1} \leq C\|\phi[\boldsymbol{x}]\|_{0} \leq C\left\|\boldsymbol{\lambda}_{h}\right\|_{0}
$$

Observe that, from (9) and (8)

$$
\begin{gathered}
\boldsymbol{v}_{h}^{(2)}(L)=\int_{0}^{L} \boldsymbol{\phi}[\boldsymbol{x}] \wedge \boldsymbol{t} d s=\boldsymbol{x}=-\boldsymbol{v}_{h}^{(1)}(L) \\
\boldsymbol{\psi}_{h}^{(2)}(0)=\boldsymbol{\psi}_{h}^{(2)}(L)=\boldsymbol{\phi}[\boldsymbol{x}](0)=\boldsymbol{\phi}[\boldsymbol{x}](L)=\mathbf{0}
\end{gathered}
$$


Next, we want to prove that this correction we add has a negligible effect through $b_{h}$ :

$$
\begin{aligned}
b_{h}\left(\boldsymbol{v}_{h}^{(2)}, \boldsymbol{\psi}_{h}^{(2)} ; \boldsymbol{\lambda}_{h}\right) & =\int_{0}^{\bar{L}} \boldsymbol{\lambda}_{h} \cdot\left(\frac{d \boldsymbol{v}_{h}^{(2)}}{d \bar{s}}-\boldsymbol{\psi}_{h}^{(2)} \wedge \overline{\boldsymbol{t}}\right) d \bar{s} \\
& =\int_{0}^{L} \boldsymbol{\lambda}_{h} \cdot\left(\frac{d \boldsymbol{v}_{h}^{(2)}}{d s} \frac{d s}{d \bar{s}}-\boldsymbol{\psi}_{h}^{(2)} \wedge \overline{\boldsymbol{t}}\right) \frac{d \bar{s}}{d s} d s \\
& \leq C\left\|\boldsymbol{\lambda}_{h}\right\|_{0}\left\|\frac{d \boldsymbol{v}_{h}^{(2)}}{d s} \frac{d s}{d \bar{s}}-\boldsymbol{\psi}_{h}^{(2)} \wedge \overline{\boldsymbol{t}}\right\|_{0} \\
& \leq C\left\|\boldsymbol{\lambda}_{h}\right\|_{0}\left\{\left\|\frac{d \boldsymbol{v}_{h}^{(2)}}{d s}-\boldsymbol{\psi}_{h}^{(2)} \wedge \boldsymbol{t}\right\|_{0}+\left\|\frac{d \boldsymbol{v}_{h}^{(2)}}{d s}\left(\frac{d s}{d \bar{s}}-1\right)\right\|_{0}\right. \\
& \leq C\left\|\boldsymbol{\lambda}_{h}\right\|_{0}\left\{\left\|\frac{d \boldsymbol{v}_{h}^{(2)}}{d s}-\boldsymbol{\phi}[\boldsymbol{x}] \wedge \boldsymbol{t}\right\|_{0}+\left\|\boldsymbol{\phi}[\boldsymbol{x}]-\boldsymbol{\psi}_{h}^{(2)}\right\|_{0}\right. \\
& \left.+\left\|\frac{d \boldsymbol{v}_{h}^{(2)}}{d s}\left(\frac{d s}{d \bar{s}}-1\right)\right\|_{0}+\left\|\boldsymbol{\psi}_{h}^{(2)} \wedge(\boldsymbol{t}-\overline{\boldsymbol{t}})\right\|_{0}\right\}
\end{aligned}
$$

The first two terms inside the braces are standard interpolation errors, so that by (10):

$$
\begin{gathered}
\left\|\frac{d \boldsymbol{v}_{h}^{(2)}}{d s}-\boldsymbol{\phi}[\boldsymbol{x}] \wedge \boldsymbol{t}\right\|_{0} \leq C h|\boldsymbol{\phi}[\boldsymbol{x}] \wedge \boldsymbol{t}|_{1} \leq C h\|\boldsymbol{x}\| \leq C h\left\|\boldsymbol{\lambda}_{h}\right\|_{0} \\
\left\|\phi[\boldsymbol{x}]-\boldsymbol{\psi}_{h}^{(2)}\right\|_{0} \leq C h|\boldsymbol{\phi}[\boldsymbol{x}]|_{1} \leq C h\|\boldsymbol{x}\| \leq C h\left\|\boldsymbol{\lambda}_{h}\right\|_{0}
\end{gathered}
$$

To bound the last two terms, we use the results of Lemma 3.1. By (32) and (43) we have:

$$
\left\|\frac{d \boldsymbol{v}_{h}^{(2)}}{d s}\left(\frac{d s}{d \bar{s}}-1\right)\right\|_{0} \leq C h^{2}\left|\boldsymbol{v}_{h}^{(2)}\right|_{1} \leq C h^{2}\left\|\boldsymbol{\lambda}_{h}\right\|_{0}
$$

while (33), (34) and (42) entail:

$$
\left\|\boldsymbol{\psi}_{h}^{(2)} \wedge(\boldsymbol{t}-\overline{\boldsymbol{t}})\right\|_{0} \leq C h\left\|\boldsymbol{\psi}_{h}^{(2)}\right\|_{0} \leq C h\left\|\boldsymbol{\lambda}_{h}\right\|_{0}
$$

RR n 2733 
Subsequently

$$
b_{h}\left(\boldsymbol{v}_{h}^{(2)}, \boldsymbol{\psi}_{h}^{(2)} ; \boldsymbol{\lambda}_{h}\right) \leq C h\left\|\boldsymbol{\lambda}_{h}\right\|_{0}^{2}
$$

Finally, we set

$$
\boldsymbol{v}_{h} \stackrel{\text { def }}{=} \boldsymbol{v}_{h}^{(1)}+\boldsymbol{v}_{h}^{(2)} \quad \boldsymbol{\psi}_{h} \stackrel{\text { def }}{=} \boldsymbol{\psi}_{h}^{(1)}+\boldsymbol{\psi}_{h}^{(2)}=\boldsymbol{\psi}_{h}^{(2)} \quad \boldsymbol{\mu}_{h} \stackrel{\text { def }}{=} \boldsymbol{\mu}_{h}^{(1)}=\mathbf{0}
$$

and we can see from (44) and (45) that our choice satisfies the homogeneous boundary conditions. Gathering (39), (42) and (43), we also have

$$
\left\|\boldsymbol{v}_{h}, \boldsymbol{\psi}_{h}\right\|_{1}+\left\|\boldsymbol{\mu}_{h}\right\|_{0} \leq C\left\|\boldsymbol{\lambda}_{h}\right\|_{0}
$$

Moreover

$$
\begin{aligned}
B_{h}\left(\boldsymbol{u}_{h}, \boldsymbol{\theta}_{h}, \boldsymbol{\lambda}_{h} ; \boldsymbol{v}_{h}, \boldsymbol{\psi}_{h}, \boldsymbol{\mu}_{h}\right) \\
\quad=B_{h}\left(\boldsymbol{u}_{h}, \boldsymbol{\theta}_{h}, \boldsymbol{\lambda}_{h} ; \boldsymbol{v}_{h}^{(1)}, \mathbf{0}, \mathbf{0}\right)+B_{h}\left(\boldsymbol{u}_{h}, \boldsymbol{\theta}_{h}, \boldsymbol{\lambda}_{h} ; \boldsymbol{v}_{h}^{(2)}, \boldsymbol{\psi}_{h}^{(2)}, \mathbf{0}\right) \\
=\left\|\boldsymbol{\lambda}_{h}\right\|_{0}^{2}+b_{h}\left(\boldsymbol{v}_{h}^{(2)}, \boldsymbol{\psi}_{h}^{(2)} ; \boldsymbol{\lambda}_{h}\right)+\int_{0}^{\bar{L}} \overline{\mathcal{J}}\left(\frac{d \boldsymbol{\theta}_{h}}{d \bar{s}}\right) \cdot \frac{d \boldsymbol{\psi}_{h}^{(2)}}{d \bar{s}} d \bar{s} \\
\geq(1-C h)\left\|\boldsymbol{\lambda}_{h}\right\|_{0}^{2}-C\left|\boldsymbol{\theta}_{h}\right|_{1}\left|\boldsymbol{\psi}_{h}^{(2)}\right|_{1} \\
\geq(1-C h)\left\|\boldsymbol{\lambda}_{h}\right\|_{0}^{2}-C\left|\boldsymbol{\theta}_{h}\right|_{1}\left\|\boldsymbol{\lambda}_{h}\right\|_{0} \\
\geq\left(\frac{1}{2}-C h\right)\left\|\boldsymbol{\lambda}_{h}\right\|_{0}^{2}-C\left|\boldsymbol{\theta}_{h}\right|_{1}^{2}
\end{aligned}
$$

Therefore, for $h$ small enough we have

$$
B_{h}\left(\boldsymbol{u}_{h}, \boldsymbol{\theta}_{h}, \boldsymbol{\lambda}_{h} ; \boldsymbol{v}_{h}, \boldsymbol{\psi}_{h}, \boldsymbol{\mu}_{h}\right) \geq C\left\|\boldsymbol{\lambda}_{h}\right\|_{0}^{2}-C\left\|\boldsymbol{\theta}_{h}\right\|_{1}^{2}
$$

iii) Stability in $\boldsymbol{u}_{h}$. We take here

$$
\boldsymbol{v}_{h} \stackrel{\text { def }}{=} \mathbf{0} \quad \boldsymbol{\psi}_{h} \stackrel{\text { def }}{=} \mathbf{0} \quad \boldsymbol{\mu}_{h} \stackrel{\text { def }}{=} \frac{d \boldsymbol{u}_{h}}{d s}
$$

Note that $\boldsymbol{\mu}_{h}$ is in $Q_{h}$. Furthermore

$$
\left\|\boldsymbol{v}_{h}, \boldsymbol{\psi}_{h}\right\|_{1}+\left\|\boldsymbol{\mu}_{h}\right\|_{0}=\left|\boldsymbol{u}_{h}\right|_{1}
$$


With this choice, we have

$$
\begin{aligned}
B_{h}\left(\boldsymbol{u}_{h}, \boldsymbol{\theta}_{h}, \boldsymbol{\lambda}_{h} ; \boldsymbol{v}_{h}, \boldsymbol{\psi}_{h}, \boldsymbol{\mu}_{h}\right) \\
\quad=\int_{0}^{\bar{L}} \boldsymbol{\mu}_{h} \cdot\left(\frac{d \boldsymbol{u}_{h}}{d \bar{s}}-\boldsymbol{\theta}_{h} \wedge \overline{\boldsymbol{t}}\right) d \bar{s}-d^{2} \int_{0}^{\bar{L}} \overline{\mathcal{A}}^{-1}\left(\boldsymbol{\lambda}_{h}\right) \cdot \boldsymbol{\mu}_{h} d \bar{s} \\
\quad=\int_{0}^{L} \boldsymbol{\mu}_{h} \cdot \frac{d \boldsymbol{u}_{h}}{d s} d s-\int_{0}^{\bar{L}} \boldsymbol{\mu}_{h} \cdot\left[\boldsymbol{\theta}_{h} \wedge \overline{\boldsymbol{t}}+d^{2} \overline{\mathcal{A}}^{-1}\left(\boldsymbol{\lambda}_{h}\right)\right] d \bar{s} \\
\geq\left|\boldsymbol{u}_{h}\right|_{1}^{2}-C\left\|\boldsymbol{\mu}_{h}\right\|_{0}\left\{\left\|\boldsymbol{\theta}_{h}\right\|_{0}+\left\|\boldsymbol{\lambda}_{h}\right\|_{0}\right\} \\
\geq C\left\|\boldsymbol{u}_{h}\right\|_{1}^{2}-C\left\{\left\|\boldsymbol{\theta}_{h}\right\|_{0}^{2}+\left\|\boldsymbol{\lambda}_{h}\right\|_{0}^{2}\right\}
\end{aligned}
$$

using (49) and the Poincaré inequality in the last transformation. Now, to conclude the stability proof, we classically invoke linear combinations of the trial functions derived at each step.

We proceed to analyse the consistency of our scheme.

Lemma 3.3 The following consistency bounds hold:

$$
\left.\begin{array}{c}
\left|B_{h}\left(\boldsymbol{u}_{h}, \boldsymbol{\theta}_{h}, \boldsymbol{\lambda}_{h} ; \boldsymbol{v}_{h}, \boldsymbol{\psi}_{h}, \boldsymbol{\mu}_{h}\right)-B\left(\boldsymbol{u}_{h}, \boldsymbol{\theta}_{h}, \boldsymbol{\lambda}_{h} ; \boldsymbol{v}_{h}, \boldsymbol{\psi}_{h}, \boldsymbol{\mu}_{h}\right)\right| \\
\leq C h^{2}\left(\left\|\boldsymbol{\theta}_{h}\right\|_{1}+\left\|\boldsymbol{\lambda}_{h}\right\|_{0}\right)\left(\left\|\boldsymbol{\psi}_{h}\right\|_{1}+\left\|\boldsymbol{\mu}_{h}\right\|_{0}\right) \\
\forall\left(\boldsymbol{u}_{h}, \boldsymbol{\theta}_{h}, \boldsymbol{\lambda}_{h}\right),\left(\boldsymbol{v}_{h}, \boldsymbol{\psi}_{h}, \boldsymbol{\mu}_{h}\right) \\
\left|F_{h}\left(\boldsymbol{v}_{h}\right)-F\left(\boldsymbol{v}_{h}\right)\right| \leq C h^{2}\|\boldsymbol{p}\|_{0}\left\|\boldsymbol{v}_{h}\right\|_{0} \quad \forall \boldsymbol{v}_{h}
\end{array}\right\}
$$

Proof: Set

$$
\begin{aligned}
\bar{B}_{h}\left(\boldsymbol{u}_{h}, \boldsymbol{\theta}_{h}, \boldsymbol{\lambda}_{h} ; \boldsymbol{v}_{h}, \boldsymbol{\psi}_{h}, \boldsymbol{\mu}_{h}\right) \stackrel{\text { def }}{=} \int_{0}^{L} \overline{\mathcal{J}}\left(\frac{d \boldsymbol{\theta}_{h}}{d s}\right) \cdot \frac{d \boldsymbol{\psi}_{h}}{d s} d s \\
+\int_{0}^{L} \boldsymbol{\lambda}_{h} \cdot\left(\frac{d \boldsymbol{v}_{h}}{d s}-\boldsymbol{\psi}_{h} \wedge \overline{\boldsymbol{t}}\right) d s \\
\quad+\int_{0}^{L} \boldsymbol{\mu}_{h} \cdot\left(\frac{d \boldsymbol{u}_{h}}{d s}-\boldsymbol{\theta}_{h} \wedge \overline{\boldsymbol{t}}\right) d s-d^{2} \int_{0}^{L} \overline{\mathcal{A}}^{-1}\left(\boldsymbol{\lambda}_{h}\right) \cdot \boldsymbol{\mu}_{h} d s
\end{aligned}
$$

RR n 2733 
We have, from (32):

$$
\begin{aligned}
& \left|B_{h}\left(\boldsymbol{u}_{h}, \boldsymbol{\theta}_{h}, \boldsymbol{\lambda}_{h} ; \boldsymbol{v}_{h}, \boldsymbol{\psi}_{h}, \boldsymbol{\mu}_{h}\right)-\bar{B}_{h}\left(\boldsymbol{u}_{h}, \boldsymbol{\theta}_{h}, \boldsymbol{\lambda}_{h} ; \boldsymbol{v}_{h}, \boldsymbol{\psi}_{h}, \boldsymbol{\mu}_{h}\right)\right| \\
& \quad=\mid \int_{0}^{L}\left(\frac{d s}{d \bar{s}}-1\right) \overline{\mathcal{J}}\left(\frac{d \boldsymbol{\theta}_{h}}{d s}\right) \cdot \frac{d \boldsymbol{\psi}_{h}}{d s} d s-\int_{0}^{L}\left(\frac{d \bar{s}}{d s}-1\right) \boldsymbol{\lambda}_{h} \cdot\left(\boldsymbol{\psi}_{h} \wedge \overline{\boldsymbol{t}}\right) d s \\
& \quad-\int_{0}^{L}\left(\frac{d \bar{s}}{d s}-1\right) \boldsymbol{\mu}_{h} \cdot\left(\boldsymbol{\theta}_{h} \wedge \overline{\boldsymbol{t}}\right) d s-d^{2} \int_{0}^{L}\left(\frac{d \bar{s}}{d s}-1\right) \overline{\mathcal{A}}^{-1}\left(\boldsymbol{\lambda}_{h}\right) \cdot \boldsymbol{\mu}_{h} d s \mid \\
& \leq C h^{2}\left(\left\|\boldsymbol{\theta}_{h}\right\|_{1}+\left\|\boldsymbol{\lambda}_{h}\right\|_{0}\right)\left(\left\|\boldsymbol{\psi}_{h}\right\|_{1}+\left\|\boldsymbol{\mu}_{h}\right\|_{0}\right)
\end{aligned}
$$

Then recall, from (26) and similar expressions:

$$
\left.\begin{array}{l}
\mathcal{J}(\boldsymbol{x}) \cdot \boldsymbol{y}=\left(j_{t}-j_{n}\right)(\boldsymbol{x} \cdot \boldsymbol{t})(\boldsymbol{y} \cdot \boldsymbol{t})+j_{n} \boldsymbol{x} \cdot \boldsymbol{y} \\
\overline{\mathcal{J}}(\boldsymbol{x}) \cdot \boldsymbol{y}=\left(j_{t}-j_{n}\right)(\boldsymbol{x} \cdot \overline{\boldsymbol{t}})(\boldsymbol{y} \cdot \overline{\boldsymbol{t}})+j_{n} \boldsymbol{x} \cdot \boldsymbol{y} \\
\mathcal{A}^{-1}(\boldsymbol{x}) \cdot \boldsymbol{y}=\left(a_{t}^{-1}-a_{n}^{-1}\right)(\boldsymbol{x} \cdot \boldsymbol{t})(\boldsymbol{y} \cdot \boldsymbol{t})+a_{n}^{-1} \boldsymbol{x} \cdot \boldsymbol{y} \\
\overline{\mathcal{A}}^{-1}(\boldsymbol{x}) \cdot \boldsymbol{y}=\left(a_{t}^{-1}-a_{n}^{-1}\right)(\boldsymbol{x} \cdot \overline{\boldsymbol{t}})(\boldsymbol{y} \cdot \overline{\boldsymbol{t}})+a_{n}^{-1} \boldsymbol{x} \cdot \boldsymbol{y}
\end{array}\right\}
$$

We infer

$$
\left.\begin{array}{rl}
\bar{B}_{h}\left(\boldsymbol{u}_{h}, \boldsymbol{\theta}_{h}, \boldsymbol{\lambda}_{h} ; \boldsymbol{v}_{h}, \boldsymbol{\psi}_{h}, \boldsymbol{\mu}_{h}\right)-B\left(\boldsymbol{u}_{h}, \boldsymbol{\theta}_{h}, \boldsymbol{\lambda}_{h} ; \boldsymbol{v}_{h}, \boldsymbol{\psi}_{h}, \boldsymbol{\mu}_{h}\right) \\
=\left(j_{t}-j_{n}\right) \int_{0}^{L}\left[\left(\frac{d \boldsymbol{\theta}_{h}}{d s} \cdot \overline{\boldsymbol{t}}\right)\left(\frac{d \boldsymbol{\psi}_{h}}{d s} \cdot \overline{\boldsymbol{t}}\right)-\left(\frac{d \boldsymbol{\theta}_{h}}{d s} \cdot \boldsymbol{t}\right)\left(\frac{d \boldsymbol{\psi}_{h}}{d s} \cdot \boldsymbol{t}\right)\right] d s \\
-\int_{0}^{L} \boldsymbol{\lambda}_{h} \cdot\left[\boldsymbol{\psi}_{h} \wedge(\overline{\boldsymbol{t}}-\boldsymbol{t})\right] d s-\int_{0}^{L} \boldsymbol{\mu}_{h} \cdot\left[\boldsymbol{\theta}_{h} \wedge(\overline{\boldsymbol{t}}-\boldsymbol{t})\right] d s \\
-d^{2}\left(a_{t}^{-1}-a_{n}^{-1}\right) \int_{0}^{L}\left[\left(\boldsymbol{\lambda}_{h} \cdot \overline{\boldsymbol{t}}\right)\left(\boldsymbol{\mu}_{h} \cdot \overline{\boldsymbol{t}}\right)-\left(\boldsymbol{\lambda}_{h} \cdot \boldsymbol{t}\right)\left(\boldsymbol{\mu}_{h} \cdot \boldsymbol{t}\right)\right] d s
\end{array}\right\}
$$


To bound the right hand side, we use (33) and (34). For the first term we have

$$
\begin{aligned}
\int_{0}^{L}\left[\left(\frac{d \boldsymbol{\theta}_{h}}{d s} \cdot \overline{\boldsymbol{t}}\right)\left(\frac{d \boldsymbol{\psi}_{h}}{d s} \cdot \overline{\boldsymbol{t}}\right)-\left(\frac{d \boldsymbol{\theta}_{h}}{d s} \cdot \boldsymbol{t}\right)\left(\frac{d \boldsymbol{\psi}_{h}}{d s} \cdot \boldsymbol{t}\right)\right] d s \\
\quad=\sum_{i=0}^{N-1} \int_{s_{i}}^{s_{i+1}}\left[\left(\frac{d \boldsymbol{\theta}_{h}}{d s} \cdot \overline{\boldsymbol{t}}\right)\left(\frac{d \boldsymbol{\psi}_{h}}{d s} \cdot \overline{\boldsymbol{t}}\right)-\left(\frac{d \boldsymbol{\theta}_{h}}{d s} \cdot \boldsymbol{t}\right)\left(\frac{d \boldsymbol{\psi}_{h}}{d s} \cdot \boldsymbol{t}\right)\right] d s \\
\quad=-\sum_{i=0}^{N-1} \int_{s_{i}}^{s_{i+1}}\left(s-s_{i+\frac{1}{2}}\right)\left[\left(\frac{d \boldsymbol{\theta}_{h}}{d s} \cdot \boldsymbol{t}\left(s_{i+\frac{1}{2}}\right)\right)\left(\frac{d \boldsymbol{\psi}_{h}}{d s} \cdot \frac{d^{2} \boldsymbol{\alpha}}{d s^{2}}\left(s_{i+\frac{1}{2}}\right)\right)\right. \\
\left.\quad+\left(\frac{d \boldsymbol{\theta}_{h}}{d s} \cdot \frac{d^{2} \boldsymbol{\alpha}}{d s^{2}}\left(s_{i+\frac{1}{2}}\right)\right)\left(\frac{d \boldsymbol{\psi}_{h}}{d s} \cdot \boldsymbol{t}\left(s_{i+\frac{1}{2}}\right)\right)\right] d s+O\left(h^{2}\right)\left\|\frac{d \boldsymbol{\theta}_{h}}{d s}\right\|_{0}\left\|\frac{d \boldsymbol{\psi}_{h}}{d s}\right\|_{0}
\end{aligned}
$$

and the integral terms vanish since $\left(s-s_{i+\frac{1}{2}}\right)$ is multiplied by a function which is constant within each $\left[s_{i}, s_{i+1}\right]$. Therefore:

$$
\left|\int_{0}^{L}\left[\left(\frac{d \boldsymbol{\theta}_{h}}{d s} \cdot \overline{\boldsymbol{t}}\right)\left(\frac{d \boldsymbol{\psi}_{h}}{d s} \cdot \overline{\boldsymbol{t}}\right)-\left(\frac{d \boldsymbol{\theta}_{h}}{d s} \cdot \boldsymbol{t}\right)\left(\frac{d \boldsymbol{\psi}_{h}}{d s} \cdot \boldsymbol{t}\right)\right] d s\right| \leq C h^{2}\left|\boldsymbol{\theta}_{h}\right|_{1}\left|\boldsymbol{\psi}_{h}\right|_{1}
$$

and an identical argument leads to

$$
\left|\int_{0}^{L}\left[\left(\boldsymbol{\lambda}_{h} \cdot \overline{\boldsymbol{t}}\right)\left(\boldsymbol{\mu}_{h} \cdot \overline{\boldsymbol{t}}\right)-\left(\boldsymbol{\lambda}_{h} \cdot \boldsymbol{t}\right)\left(\boldsymbol{\mu}_{h} \cdot \boldsymbol{t}\right)\right] d s\right| \leq C h^{2}\left\|\boldsymbol{\lambda}_{h}\right\|_{0}\left\|\boldsymbol{\mu}_{h}\right\|_{0}
$$

Moreover

$$
\begin{aligned}
\int_{0}^{L} \boldsymbol{\lambda}_{h} \cdot\left[\boldsymbol{\psi}_{h} \wedge(\overline{\boldsymbol{t}}-\boldsymbol{t})\right] d s \\
=-\sum_{i=0}^{N-1} \int_{s_{i}}^{s_{i+1}}\left(s-s_{i+\frac{1}{2}}\right) \boldsymbol{\lambda}_{h} \cdot\left(\boldsymbol{\psi}_{h} \wedge \frac{d^{2} \boldsymbol{\alpha}}{d s^{2}}\left(s_{i+\frac{1}{2}}\right)\right) d s+O\left(h^{2}\right)\left\|\boldsymbol{\lambda}_{h}\right\|_{0}\left\|\boldsymbol{\psi}_{h}\right\|_{0} \\
=-\sum_{i=0}^{N-1} \int_{s_{i}}^{s_{i+1}}\left(s-s_{i+\frac{1}{2}}\right)^{2} \boldsymbol{\lambda}_{h} \cdot\left(\frac{d \boldsymbol{\psi}_{h}}{d s} \wedge \frac{d^{2} \boldsymbol{\alpha}}{d s^{2}}\left(s_{i+\frac{1}{2}}\right)\right) d s+O\left(h^{2}\right)\left\|\boldsymbol{\lambda}_{h}\right\|_{0}\left\|\boldsymbol{\psi}_{h}\right\|_{0}
\end{aligned}
$$

since $\boldsymbol{\psi}_{h}$ is piecewise-linear, while $\boldsymbol{\lambda}_{h}$ and $\frac{d^{2} \boldsymbol{\alpha}}{d s^{2}}\left(s_{i+\frac{1}{2}}\right)$ are piecewise-constant. Thus:

$$
\left|\int_{0}^{L} \boldsymbol{\lambda}_{h} \cdot\left[\boldsymbol{\psi}_{h} \wedge(\overline{\boldsymbol{t}}-\boldsymbol{t})\right] d s\right| \leq C h^{2}\left\|\boldsymbol{\lambda}_{h}\right\|_{0}\left\|\boldsymbol{\psi}_{h}\right\|_{1}
$$

RR n 2733 
Subsequently, gathering (54), (55), (56) and (57) we obtain

$$
\left.\begin{array}{r}
\left|\bar{B}_{h}\left(\boldsymbol{u}_{h}, \boldsymbol{\theta}_{h}, \boldsymbol{\lambda}_{h} ; \boldsymbol{v}_{h}, \boldsymbol{\psi}_{h}, \boldsymbol{\mu}_{h}\right)-B\left(\boldsymbol{u}_{h}, \boldsymbol{\theta}_{h}, \boldsymbol{\lambda}_{h} ; \boldsymbol{v}_{h}, \boldsymbol{\psi}_{h}, \boldsymbol{\mu}_{h}\right)\right| \\
\leq C h^{2}\left(\left\|\boldsymbol{\theta}_{h}\right\|_{1}+\left\|\boldsymbol{\lambda}_{h}\right\|_{0}\right)\left(\left\|\boldsymbol{\psi}_{h}\right\|_{1}+\left\|\boldsymbol{\mu}_{h}\right\|_{0}\right)
\end{array}\right\}
$$

which, combined with (52), yields (50). Finally

$$
F_{h}\left(\boldsymbol{v}_{h}\right)-F\left(\boldsymbol{v}_{h}\right)=\int_{0}^{L}\left(\frac{d \bar{s}}{d s}-1\right) \boldsymbol{p} \cdot \boldsymbol{v}_{h} d s
$$

and (51) immediately follows from (32).

We are now in a position to establish optimal error estimates.

Theorem 3.1 Problem $\left(\mathcal{P}_{d, h}^{\prime}\right)$ has a unique solution $\left(\boldsymbol{u}_{h}, \boldsymbol{\theta}_{h}, \boldsymbol{\lambda}_{h}\right)$ in $V_{h} \times Q_{h}$, and this solution satisfies the following error estimate:

$$
\left\|\boldsymbol{u}-\boldsymbol{u}_{h}, \boldsymbol{\theta}-\boldsymbol{\theta}_{h}\right\|_{1}+\left\|\boldsymbol{\lambda}-\boldsymbol{\lambda}_{h}\right\|_{0} \leq C h\|\boldsymbol{p}\|_{0}
$$

Proof: That $\left(\mathcal{P}_{d, h}^{\prime}\right)$ admits a unique solution is a simple consequence of the stability property of Lemma 3.2. Let now $\left(\boldsymbol{u}_{h}^{0}, \boldsymbol{\theta}_{h}^{0}, \boldsymbol{\lambda}_{h}^{0}\right)$ be any element of $V_{h} \times Q_{h}$. Lemma 3.2 ensures that one can find $\left(\boldsymbol{v}_{h}, \boldsymbol{\psi}_{h}, \boldsymbol{\mu}_{h}\right)$ in $V_{h} \times Q_{h}$ such that

$$
\left.\begin{array}{c}
\left\|\boldsymbol{v}_{h}, \boldsymbol{\psi}_{h}\right\|_{1}+\left\|\boldsymbol{\mu}_{h}\right\|_{0} \leq\left\|\boldsymbol{u}_{h}-\boldsymbol{u}_{h}^{0}, \boldsymbol{\theta}_{h}-\boldsymbol{\theta}_{h}^{0}\right\|_{1}+\left\|\boldsymbol{\lambda}_{h}-\boldsymbol{\lambda}_{h}^{0}\right\|_{0} \\
B_{h}\left(\boldsymbol{u}_{h}-\boldsymbol{u}_{h}^{0}, \boldsymbol{\theta}_{h}-\boldsymbol{\theta}_{h}^{0}, \boldsymbol{\lambda}_{h}-\boldsymbol{\lambda}_{h}^{0} ; \boldsymbol{v}_{h}, \boldsymbol{\psi}_{h}, \boldsymbol{\mu}_{h}\right) \\
\geq C\left\{\left\|\boldsymbol{u}_{h}-\boldsymbol{u}_{h}^{0}, \boldsymbol{\theta}_{h}-\boldsymbol{\theta}_{h}^{0}\right\|_{1}+\left\|\boldsymbol{\lambda}_{h}-\boldsymbol{\lambda}_{h}^{0}\right\|_{0}\right\}^{2}
\end{array}\right\}
$$

Making use of (31) and (4) we have 


$$
\left.\begin{array}{l}
B_{h}\left(\boldsymbol{u}_{h}-\boldsymbol{u}_{h}^{0}, \boldsymbol{\theta}_{h}-\boldsymbol{\theta}_{h}^{0}, \boldsymbol{\lambda}_{h}-\boldsymbol{\lambda}_{h}^{0} ; \boldsymbol{v}_{h}, \boldsymbol{\psi}_{h}, \boldsymbol{\mu}_{h}\right) \\
\quad=F_{h}\left(\boldsymbol{v}_{h}\right)-F\left(\boldsymbol{v}_{h}\right) \\
\quad+B\left(\boldsymbol{u}_{h}^{0}, \boldsymbol{\theta}_{h}^{0}, \boldsymbol{\lambda}_{h}^{0} ; \boldsymbol{v}_{h}, \boldsymbol{\psi}_{h}, \boldsymbol{\mu}_{h}\right)-B_{h}\left(\boldsymbol{u}_{h}^{0}, \boldsymbol{\theta}_{h}^{0}, \boldsymbol{\lambda}_{h}^{0} ; \boldsymbol{v}_{h}, \boldsymbol{\psi}_{h}, \boldsymbol{\mu}_{h}\right) \\
\quad+B\left(\boldsymbol{u}-\boldsymbol{u}_{h}^{0}, \boldsymbol{\theta}-\boldsymbol{\theta}_{h}^{0}, \boldsymbol{\lambda}-\boldsymbol{\lambda}_{h}^{0} ; \boldsymbol{v}_{h}, \boldsymbol{\psi}_{h}, \boldsymbol{\mu}_{h}\right) \\
\quad \leq C\left\{h^{2}\left(\|\boldsymbol{p}\|_{0}+\left\|\boldsymbol{\theta}_{h}^{0}\right\|_{1}+\left\|\boldsymbol{\lambda}_{h}^{0}\right\|_{0}\right)\right. \\
\left.\quad+\left\|\boldsymbol{u}-\boldsymbol{u}_{h}^{0}, \boldsymbol{\theta}-\boldsymbol{\theta}_{h}^{0}\right\|_{1}+\left\|\boldsymbol{\lambda}-\boldsymbol{\lambda}_{h}^{0}\right\|_{0}\right\}\left\{\left\|\boldsymbol{v}_{h}, \boldsymbol{\psi}_{h}\right\|_{1}+\left\|\boldsymbol{\mu}_{h}\right\|_{0}\right\}
\end{array}\right\}
$$

where the bound was derived by using the consistency results (50)(51) and CauchySchwarz. We then specialize $\left(\boldsymbol{u}_{h}^{0}, \boldsymbol{\theta}_{h}^{0}, \boldsymbol{\lambda}_{h}^{0}\right)$ by taking $\left(\boldsymbol{u}_{h}^{0}, \boldsymbol{\theta}_{h}^{0}\right)$ as the linear interpolant of $(\boldsymbol{u}, \boldsymbol{\theta})$ and $\boldsymbol{\lambda}_{h}$ as the $L^{2}$-projection of $\boldsymbol{\lambda}$ onto piecewise-constant functions. Applying Proposition 2.3, this choice entails:

$$
\left\|\boldsymbol{u}-\boldsymbol{u}_{h}^{0}, \boldsymbol{\theta}-\boldsymbol{\theta}_{h}^{0}\right\|_{1}+\left\|\boldsymbol{\lambda}-\boldsymbol{\lambda}_{h}^{0}\right\|_{0} \leq C h\left\{\|\boldsymbol{u}, \boldsymbol{\theta}\|_{2}+\|\boldsymbol{\lambda}\|_{1}\right\} \leq C h\|\boldsymbol{p}\|_{0}
$$

and also

$$
\left\|\boldsymbol{u}_{h}^{0}, \boldsymbol{\theta}_{h}^{0}\right\|_{1}+\left\|\boldsymbol{\lambda}_{h}^{0}\right\|_{0} \leq C\left\{\|\boldsymbol{u}, \boldsymbol{\theta}\|_{1}+\|\boldsymbol{\lambda}\|_{0}\right\} \leq C\|\boldsymbol{p}\|_{0}
$$

Therefore, combining (60), (61) and (62) we obtain

$$
\left\|\boldsymbol{u}_{h}-\boldsymbol{u}_{h}^{0}, \boldsymbol{\theta}_{h}-\boldsymbol{\theta}_{h}^{0}\right\|_{1}+\left\|\boldsymbol{\lambda}_{h}-\boldsymbol{\lambda}_{h}^{0}\right\|_{0} \leq C h\|\boldsymbol{p}\|_{0}
$$

and (59) follows by the triangle inequality.

Finally, we prove higher order $L^{2}$-error estimates for the displacements.

Theorem 3.2 The following estimate holds

$$
\left\|\boldsymbol{u}-\boldsymbol{u}_{h}, \boldsymbol{\theta}-\boldsymbol{\theta}_{h}\right\|_{0} \leq C h^{2}\|\boldsymbol{p}\|_{0}
$$

Proof: We adapt the classic duality argument of Aubin-Nitsche. Consider the problem: find $(\boldsymbol{w}, \boldsymbol{\eta}, \boldsymbol{\nu})$ in $V \times Q$ such that

$$
B(\boldsymbol{v}, \boldsymbol{\psi}, \boldsymbol{\mu} ; \boldsymbol{w}, \boldsymbol{\eta}, \boldsymbol{\nu})=\left(\boldsymbol{u}-\boldsymbol{u}_{h}, \boldsymbol{v}\right)+\left(\boldsymbol{\theta}-\boldsymbol{\theta}_{h}, \boldsymbol{\psi}\right) \quad \forall(\boldsymbol{v}, \boldsymbol{\psi}, \boldsymbol{\mu}) \in V \times Q
$$


From Proposition 2.3 we know that this problem has a unique solution and that this solution satisfies

$$
\|\boldsymbol{w}, \boldsymbol{\eta}\|_{2}+\|\boldsymbol{\nu}\|_{1} \leq C\left\{\left\|\boldsymbol{u}-\boldsymbol{u}_{h}\right\|_{0}+\left\|\boldsymbol{\theta}-\boldsymbol{\theta}_{h}\right\|_{0}\right\}
$$

Taking $(\boldsymbol{v}, \boldsymbol{\psi}, \boldsymbol{\mu})=\left(\boldsymbol{u}-\boldsymbol{u}_{h}, \boldsymbol{\theta}-\boldsymbol{\theta}_{h}, \boldsymbol{\lambda}-\boldsymbol{\lambda}_{h}\right)$ in (67) we have

$$
\begin{aligned}
\| \boldsymbol{u}- & \boldsymbol{u}_{h}\left\|_{0}^{2}+\right\| \boldsymbol{\theta}-\boldsymbol{\theta}_{h} \|_{0}^{2}=B\left(\boldsymbol{u}-\boldsymbol{u}_{h}, \boldsymbol{\theta}-\boldsymbol{\theta}_{h}, \boldsymbol{\lambda}-\boldsymbol{\lambda}_{h} ; \boldsymbol{w}, \boldsymbol{\eta}, \boldsymbol{\nu}\right) \\
\quad= & B\left(\boldsymbol{u}-\boldsymbol{u}_{h}, \boldsymbol{\theta}-\boldsymbol{\theta}_{h}, \boldsymbol{\lambda}-\boldsymbol{\lambda}_{h} ; \boldsymbol{w}-\boldsymbol{w}_{h}, \boldsymbol{\eta}-\boldsymbol{\eta}_{h}, \boldsymbol{\nu}-\boldsymbol{\nu}_{h}\right) \\
& +F\left(\boldsymbol{w}_{h}\right)-F_{h}\left(\boldsymbol{w}_{h}\right)+B_{h}\left(\boldsymbol{u}_{h}, \boldsymbol{\theta}_{h}, \boldsymbol{\lambda}_{h} ; \boldsymbol{w}_{h}, \boldsymbol{\eta}_{h}, \boldsymbol{\nu}_{h}\right)-B\left(\boldsymbol{u}_{h}, \boldsymbol{\theta}_{h}, \boldsymbol{\lambda}_{h} ; \boldsymbol{w}_{h}, \boldsymbol{\eta}_{h}, \boldsymbol{\nu}_{h}\right)
\end{aligned}
$$

for any $\left(\boldsymbol{w}_{h}, \boldsymbol{\eta}_{h}, \boldsymbol{\nu}_{h}\right)$ in $V_{h} \times Q_{h}$. By Cauchy-Schwarz, (50) and (51) we infer

$$
\begin{aligned}
& \| \boldsymbol{u}- \boldsymbol{u}_{h}\left\|_{0}^{2}+\right\| \boldsymbol{\theta}-\boldsymbol{\theta}_{h} \|_{0}^{2} \leq C\left\{\left\|\boldsymbol{u}-\boldsymbol{u}_{h}, \boldsymbol{\theta}-\boldsymbol{\theta}_{h}\right\|_{1}+\left\|\boldsymbol{\lambda}-\boldsymbol{\lambda}_{h}\right\|_{0}\right\} \\
& \times\left\{\left\|\boldsymbol{w}-\boldsymbol{w}_{h}, \boldsymbol{\eta}-\boldsymbol{\eta}_{h}\right\|_{1}+\left\|\boldsymbol{\nu}-\boldsymbol{\nu}_{h}\right\|_{0}\right\} \\
&+ C h^{2}\left\{\|\boldsymbol{p}\|_{0}+\left\|\boldsymbol{u}_{h}, \boldsymbol{\theta}_{h}\right\|_{1}+\left\|\boldsymbol{\lambda}_{h}\right\|_{0}\right\}\left\{\left\|\boldsymbol{w}_{h}, \boldsymbol{\eta}_{h}\right\|_{1}+\left\|\boldsymbol{\nu}_{h}\right\|_{0}\right\} \\
& \leq C h\|\boldsymbol{p}\|_{0}\left\{\left\|\boldsymbol{w}-\boldsymbol{w}_{h}, \boldsymbol{\eta}-\boldsymbol{\eta}_{h}\right\|_{1}+\left\|\boldsymbol{\nu}-\boldsymbol{\nu}_{h}\right\|_{0}\right\} \\
&+C h^{2}\|\boldsymbol{p}\|_{0}\left\{\left\|\boldsymbol{w}_{h}, \boldsymbol{\eta}_{h}\right\|_{1}+\left\|\boldsymbol{\nu}_{h}\right\|_{0}\right\}
\end{aligned}
$$

Now specialize $\left(\boldsymbol{w}_{h}, \boldsymbol{\eta}_{h}, \boldsymbol{\nu}_{h}\right)$ by taking $\left(\boldsymbol{w}_{h}, \boldsymbol{\eta}_{h}\right)$ as the interpolant of $(\boldsymbol{w}, \boldsymbol{\eta})$ and $\boldsymbol{\nu}_{h}$ as the $L^{2}$-projection of $\boldsymbol{\nu}$ so that

$$
\left.\begin{array}{rl}
\left\|\boldsymbol{w}-\boldsymbol{w}_{h}, \boldsymbol{\eta}-\boldsymbol{\eta}_{h}\right\|_{1}+\left\|\boldsymbol{\nu}-\boldsymbol{\nu}_{h}\right\|_{0} & \leq C h\left\{\|\boldsymbol{w}, \boldsymbol{\eta}\|_{2}+\|\boldsymbol{\nu}\|_{1}\right\} \\
& \leq C h\left\{\left\|\boldsymbol{u}-\boldsymbol{u}_{h}\right\|_{0}+\left\|\boldsymbol{\theta}-\boldsymbol{\theta}_{h}\right\|_{0}\right\}
\end{array}\right\}
$$

from (68), and also

$$
\left\|\boldsymbol{w}_{h}, \boldsymbol{\eta}_{h}\right\|_{1}+\left\|\boldsymbol{\nu}_{h}\right\|_{0} \leq C\left\{\|\boldsymbol{w}, \boldsymbol{\eta}\|_{1}+\|\boldsymbol{\nu}\|_{0}\right\} \leq C\left\{\left\|\boldsymbol{u}-\boldsymbol{u}_{h}\right\|_{0}+\left\|\boldsymbol{\theta}-\boldsymbol{\theta}_{h}\right\|_{0}\right\}
$$


Therefore, collecting (69), (70) and (71), we can write

$$
\begin{aligned}
\left\{\left\|\boldsymbol{u}-\boldsymbol{u}_{h}\right\|_{0}+\left\|\boldsymbol{\theta}-\boldsymbol{\theta}_{h}\right\|_{0}\right\}^{2} & \leq 2\left\{\left\|\boldsymbol{u}-\boldsymbol{u}_{h}\right\|_{0}^{2}+\left\|\boldsymbol{\theta}-\boldsymbol{\theta}_{h}\right\|_{0}^{2}\right\} \\
& \leq C h^{2}\|\boldsymbol{p}\|_{0}\left\{\left\|\boldsymbol{u}-\boldsymbol{u}_{h}\right\|_{0}+\left\|\boldsymbol{\theta}-\boldsymbol{\theta}_{h}\right\|_{0}\right\}
\end{aligned}
$$

and estimate (66) follows.

\section{Numerical experiments}

In this section, we demonstrate the numerical behaviour of the proposed scheme using two specific examples where closed-form solutions can be obtained. Our main goal is to investigate in practice the sensitivity of the method to the thickness, therefore we will plot error graphs for different values of $d$. In both cases, $\mathcal{A}$ and $\mathcal{J}$ are taken as the identity operators. The approximate solutions are obtained by dividing the line of centroids into $N$ elements of equal length.

\subsection{Helix}

This example is borrowed from [3]. The centroidal line is given by

$$
\boldsymbol{\alpha}(s)=\left(\cos \frac{s}{\sqrt{2}}, \sin \frac{s}{\sqrt{2}}, \frac{s}{\sqrt{2}}\right) \quad s \in\left[-\frac{\pi}{\sqrt{2}},+\frac{\pi}{\sqrt{2}}\right]
$$

The rod is clamped at both ends and the load is a uniformly distributed force directed along $\boldsymbol{z}$ (e.g. the weight, see Figure 2).

The relative errors are plotted in Figures 3 and 4 for the $L^{2}$-norm and $H^{1}$ semi-norm, and for various values of $d\left(10^{-1}, 10^{-2}, 10^{-3}\right)$. The graphs display no distinguishable difference between results obtained for different values of $d$. Furthermore, they show that convergence occurs at the expected rates, i.e. linear in $H^{1}$ and quadratic in $L^{2}$.

\subsection{Semicircular arch}

We consider here the semicircular arch described by

$$
\boldsymbol{\alpha}(s)=(\cos s, 0, \sin s) \quad s \in[0, \pi]
$$

RR n'2733 


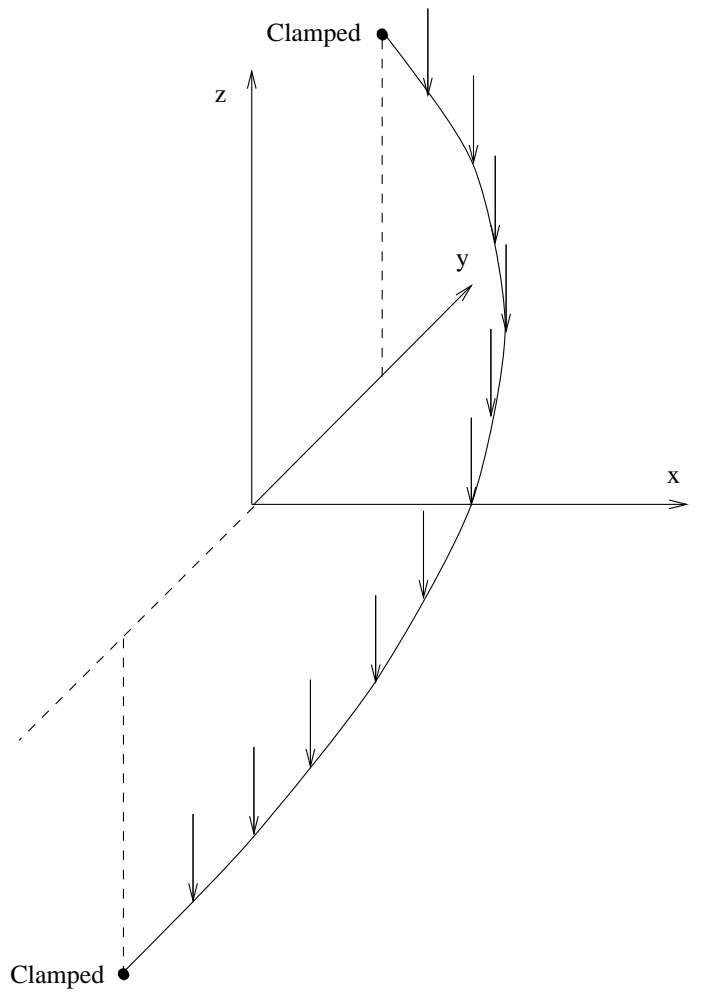

Figure 2: Helix under uniform load 


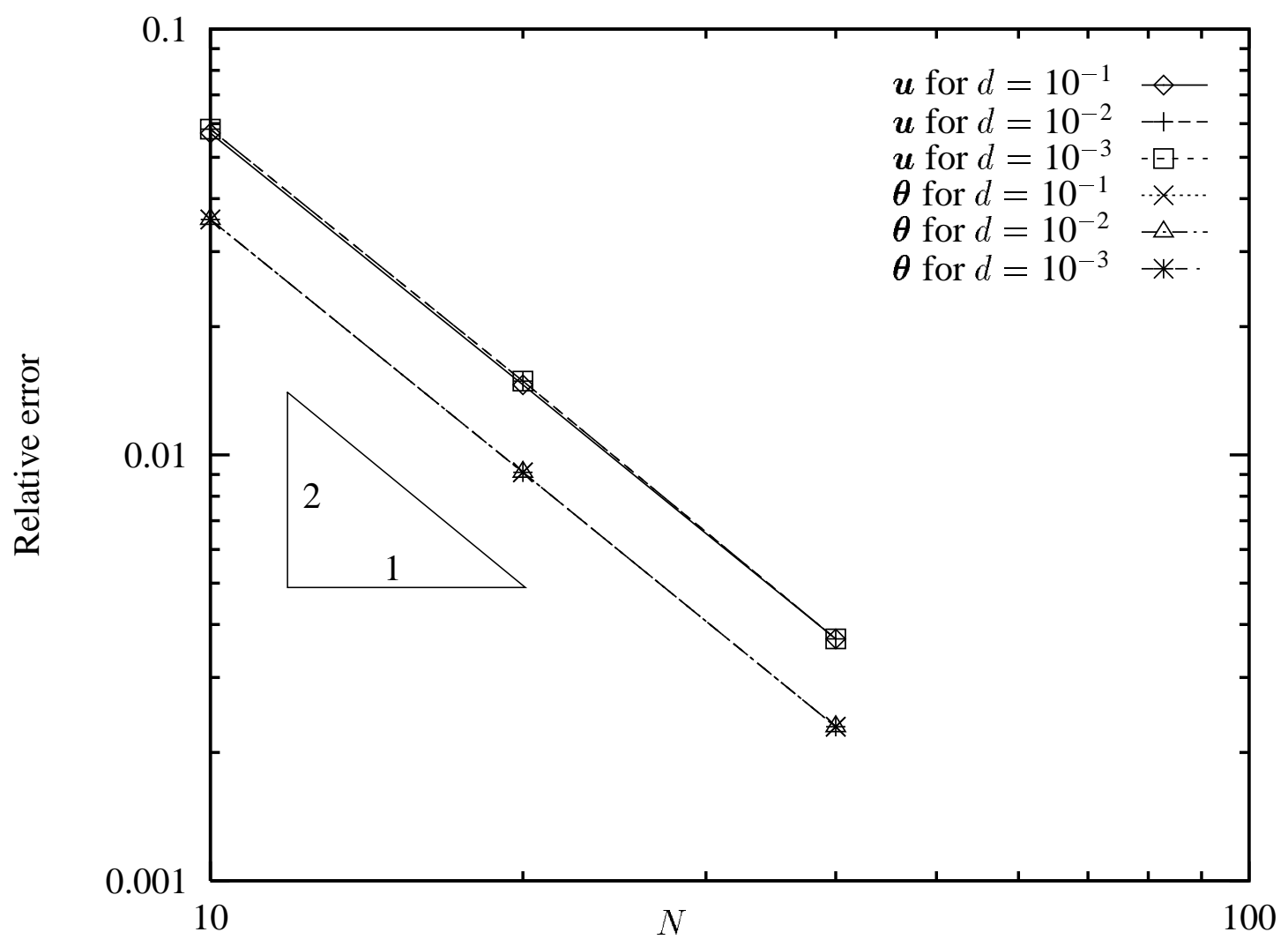

Figure 3: Helix. Errors in $L^{2}$-norm

RR n 2733 


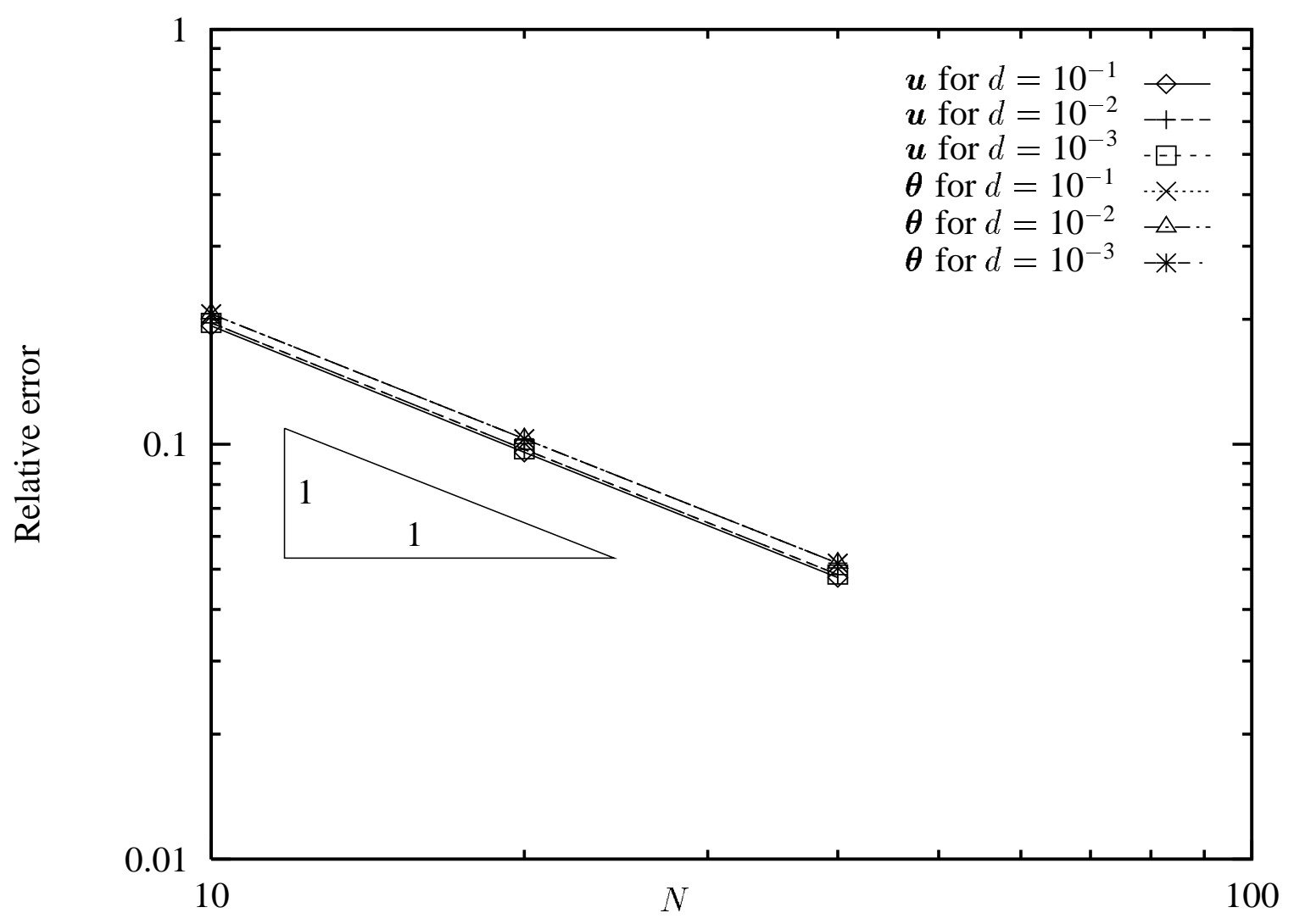

Figure 4: Helix. Errors in $H^{1}$-semi-norm 


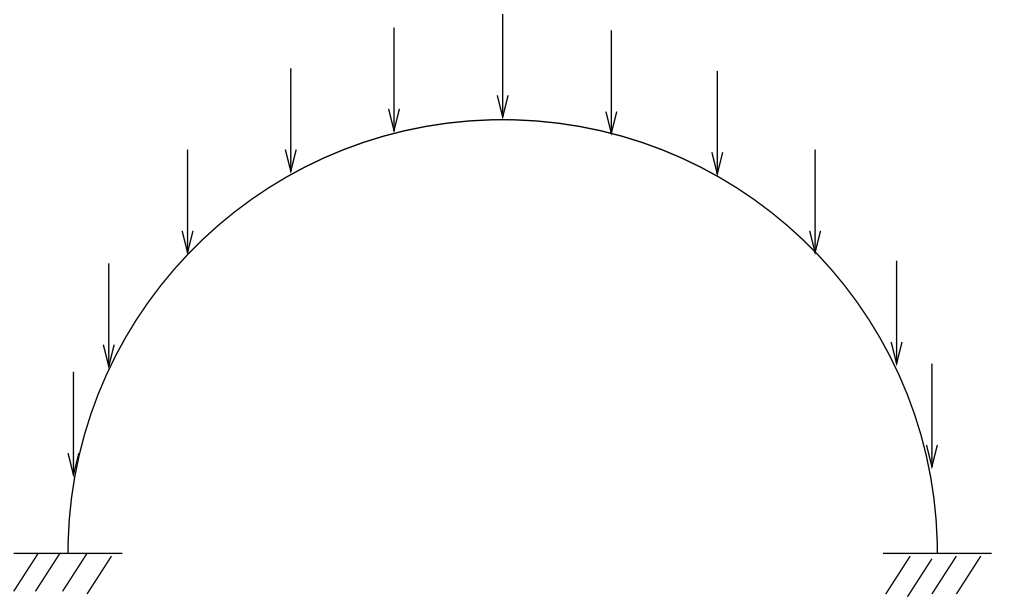

Figure 5: Arch under uniform load

clamped at both ends, and loaded by a uniform distributed force directed along $z$ (see Figure 5).

The corresponding errors are plotted in Figures 6 and 7. Again, convergence rates are in perfect agreement with theoretical results. However, the graphs now display a deterioration of the convergence behaviour when the thickness diminishes. This is particularly noticeable for $\boldsymbol{u}$, for which the $H^{1}$-error is multiplied by a factor close to 2 between $d=10^{-1}$ and $d=10^{-3}$. But it is important to note that this deterioration is visibly limited, as the curves obtained for $d=10^{-2}$ and $d=10^{-3}$ are already hardly distinguishable.

This is confirmed by Figure 8 that plots the $H^{1}$-errors against the thickness for 40 elements. It shows that the deterioration of convergence is stabilized for $d$ smaller than $10^{-2}$. This same figure also displays the errors for a numerical scheme similar to the one we described up to the fact that no projection is used in the shear/membrane term. We know that this scheme is subject to shear locking when the structure in consideration is a straight beam [1], and we expect locking to arise in our example too. This clearly appears in the numerical results, which are close to those obtained by the scheme we propose for $d$ close to unity but exhibit increasing errors as $d$ gets smaller. This deterioration encounters no limit and exceeds $99 \%$ (vanishing approximate solution) for $d=10^{-3}$, in dramatic contrast with the previous results. 


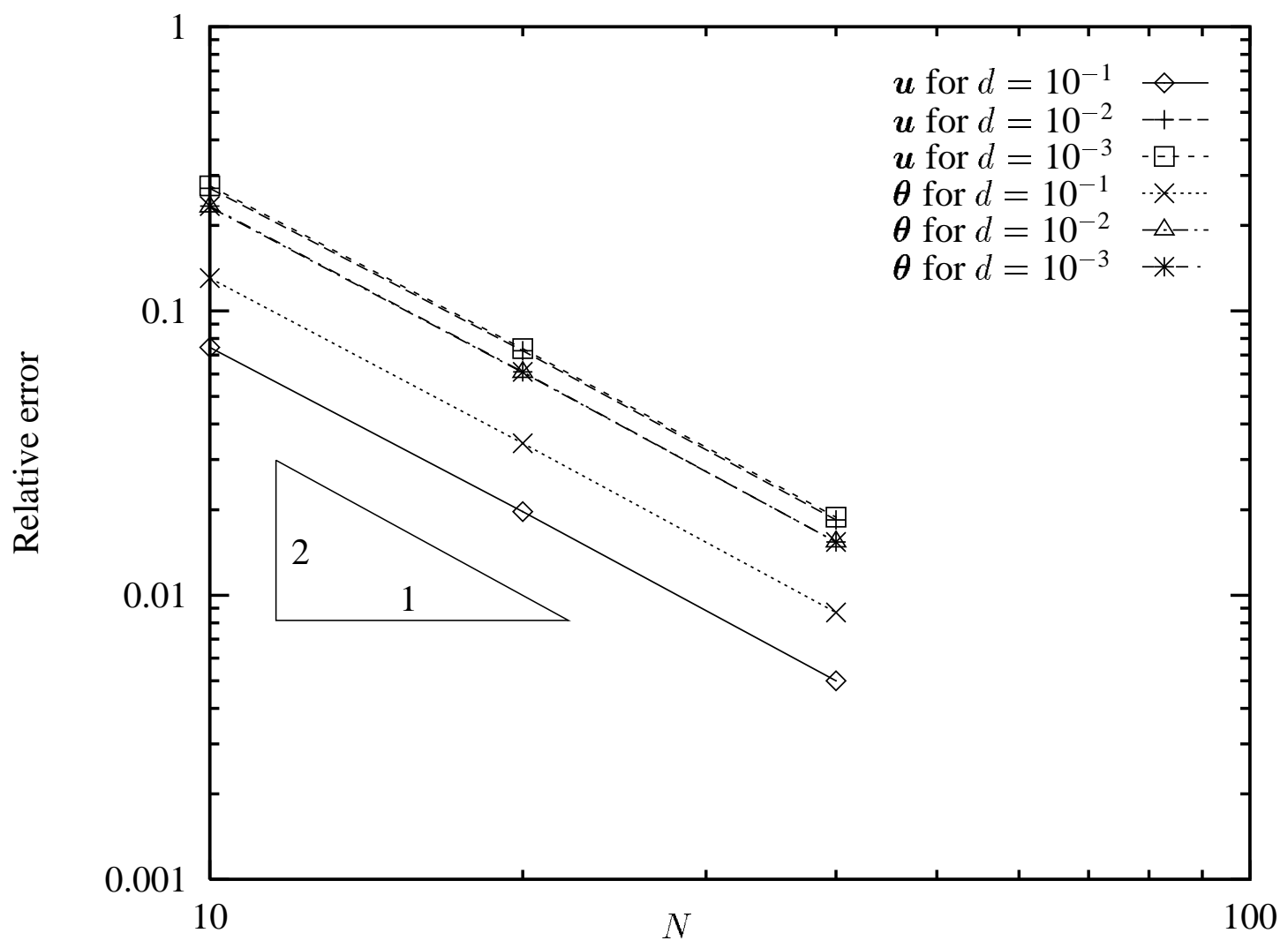

Figure 6: Semicircular arch. Errors in $L^{2}$-norm 


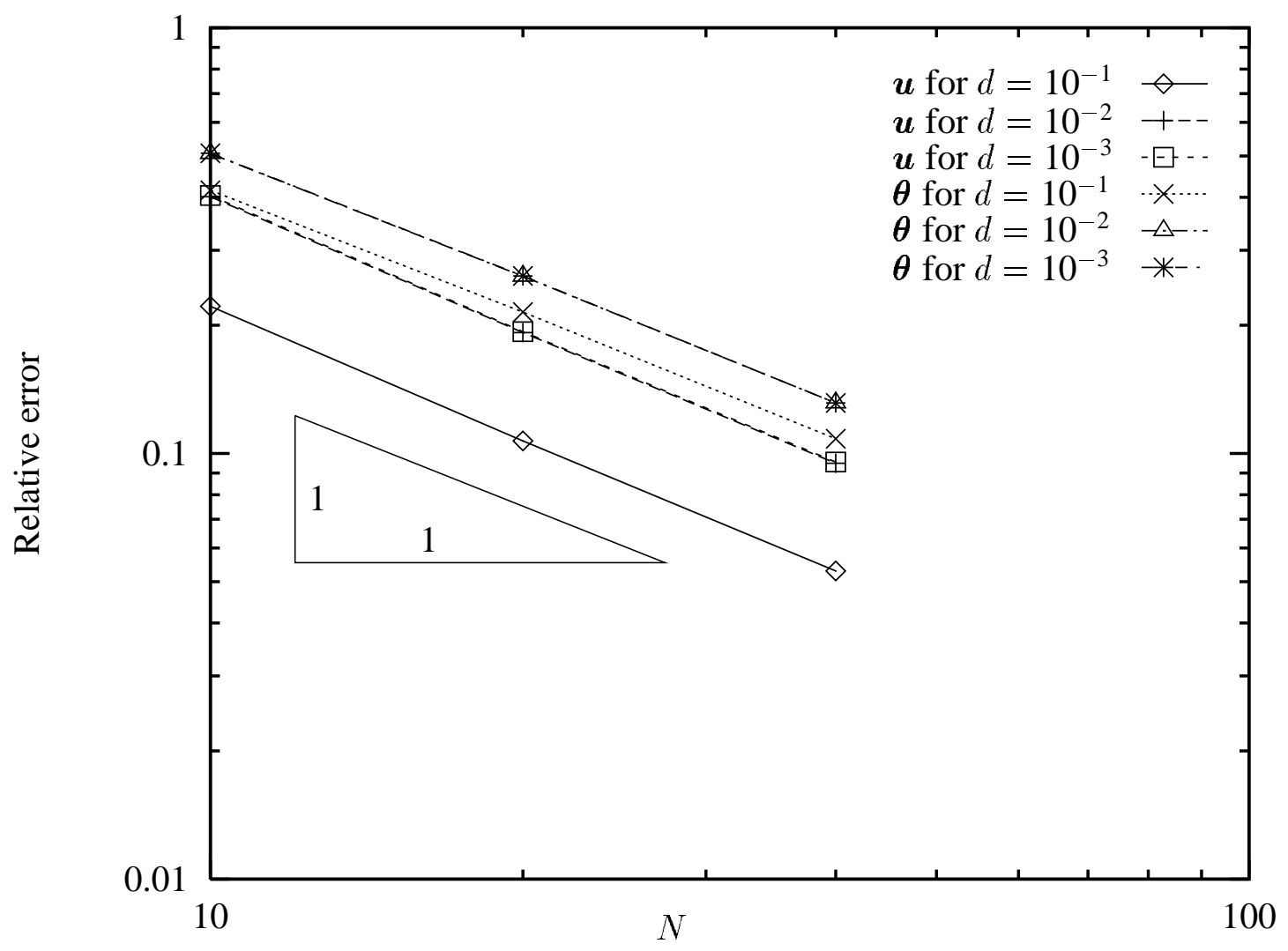

Figure 7: Semicircular arch. Errors in $H^{1}$-semi-norm

RR n²733 


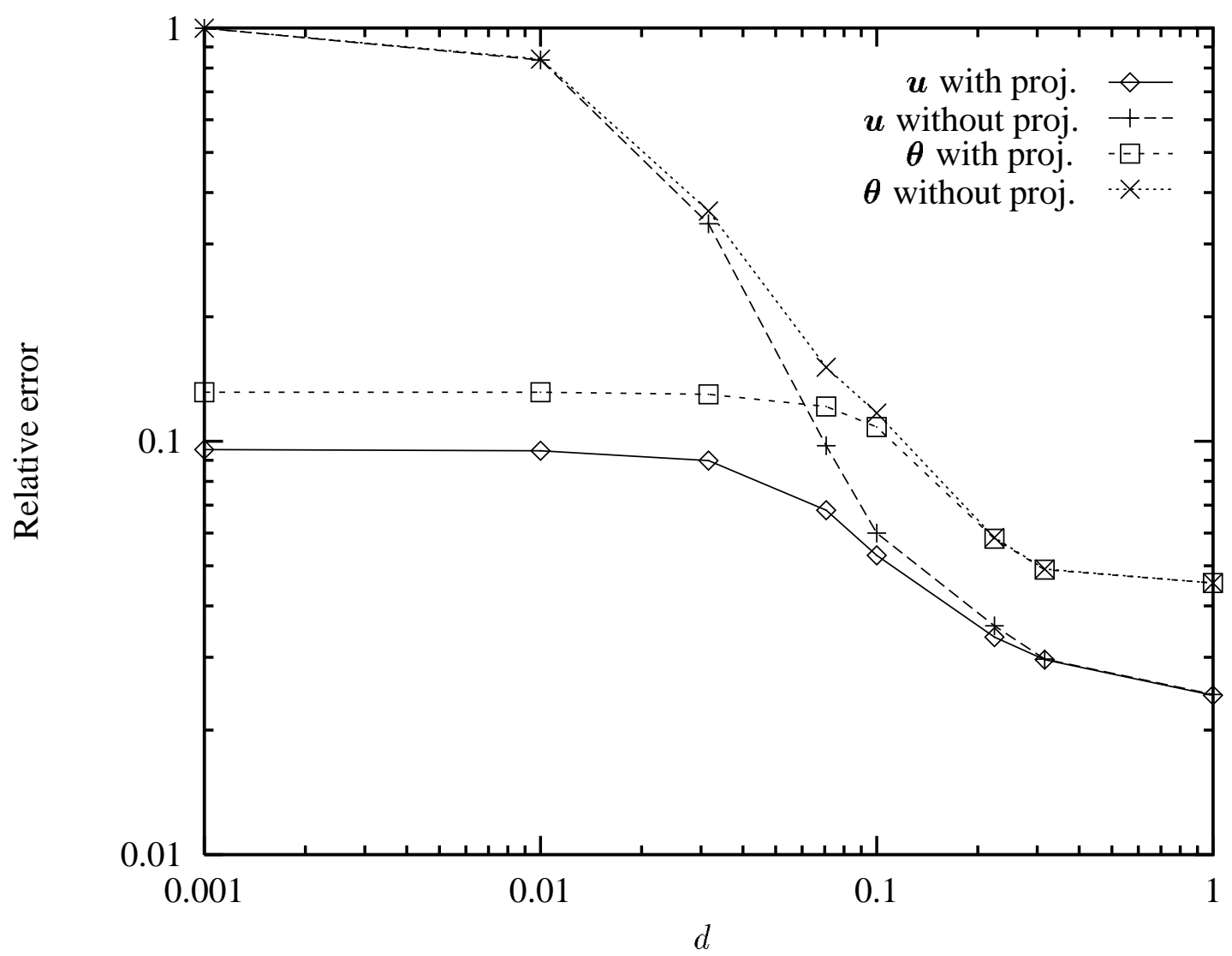

Figure 8: $H^{1}$-errors with and without projection. $N=40$ 
Thus, while this example teaches that our approximation technique does not completely rule out sensitivity of the convergence with respect to the thickness, it shows that this phenomenon is cirscumscribed within reasonable bounds, so that locking is avoided.

\section{Acknowledgement}

The author expresses his gratitude to Pr Michel Bernadou for suggesting this area of investigation and for many helpful discussions.

\section{References}

[1] D.N. Arnold. Discretization by finite elements of a model parameter dependent problem. Numer. Math., 37:405-421, 1981.

[2] D.N. Arnold and F. Brezzi. Locking free finite elements for shells. Technical Report 898, Istituto di Analisi Numerica, Pavia, 1993.

[3] K. Arunakirinathar and B. D. Reddy. Mixed finite element methods for elastic rods of arbitrary geometry. Numer. Math., 64:13-43, 1993.

[4] K.J. Bathe. Finite Element Procedures. Prentice-Hall, 1995.

[5] M. Bernadou and Y. Ducatel. Approximation of general arch problems by straight beam elements. Numer. Math., 40:1-29, 1982.

[6] F. Brezzi and M. Fortin. Mixed and Hybrid Finite Element Methods. SpringerVerlag, 1991.

[7] D. Chenais and J.-C. Paumier. On the locking phenomenon for a class of elliptic problems. Numer. Math., 67:427-440, 1994.

[8] P.G. Ciarlet. The Finite Element Methodfor Elliptic Problems. North-Holland, 1978.

RR n 2733 
[9] F. Kikuchi. On the validity of the finite element analysis of circular arches represented by an assemblage of straight beam elements. Comput. Methods Appl. Mech. Engrg., 5:253-276, 1975.

[10] F. Kikuchi. Accuracy of some finite element models for arch problems. Comput. Methods Appl. Mech. Engrg., 35:315-345, 1982.

[11] F. Kikuchi and K. Tanizawa. Accuracy and locking-free property of the beam element approximation for arch problems. Comput. \& Structures, 19(1-2):103$110,1984$.

[12] A.F.D. Loula, L.P. Franca, T.J.R. Hughes, and I. Miranda. Stability, convergence and accuracy of a new finite element method for the circular arch problem. Comput. Methods Appl. Mech. Engrg., 63:281-303, 1987.

[13] H.C. Martin. Introduction to Matrix Methods of Structural Analysis. McGrawHill, 1966.

[14] J. Pitkäranta. The problem of membrane locking in finite element analysis of cylindrical shells. Numer. Math., 61:523-542, 1992.

[15] B.D. Reddy and M.B. Volpi. Mixed finite element methods for the circular arch problem. Comput. Methods Appl. Mech. Engrg., 97:125-145, 1992. 


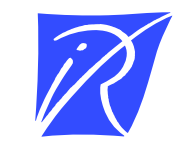

Unité de recherche INRIA Lorraine, Technopôle de Nancy-Brabois, Campus scientifique, 615 rue du Jardin Botanique, BP 101, 54600 VILLERS LES NANCY

Unité de recherche INRIA Rennes, Irisa, Campus universitaire de Beaulieu, 35042 RENNES Cedex

Unité de recherche INRIA Rhône-Alpes, 46 avenue Félix Viallet, 38031 GRENOBLE Cedex 1

Unité de recherche INRIA Rocquencourt, Domaine de Voluceau, Rocquencourt, BP 105, 78153 LE CHESNAY Cedex

Unité de recherche INRIA Sophia-Antipolis, 2004 route des Lucioles, BP 93, 06902 SOPHIA-ANTIPOLIS Cedex

Éditeur

INRIA, Domaine de Voluceau, Rocquencourt, BP 105, 78153 LE CHESNAY Cedex (France)

ISSN 0249-6399 